\title{
A charged membrane paradigm at large D
}

\author{
Sayantani Bhattacharyya, ${ }^{a}$ Mangesh Mandlik, ${ }^{b}$ Shiraz Minwalla ${ }^{b}$ \\ and Somyadip Thakur ${ }^{b}$ \\ ${ }^{a}$ Indian Institute of Technology Kanpur, \\ Kanpur, 208016 India \\ ${ }^{b}$ Tata Institute of Fundamental Research, \\ Mumbai, 400005 India \\ E-mail: sayanta@iitk.ac.in, mangesh@theory.tifr.res.in, \\ minwalla@theory.tifr.res.in, somyadip@theory.tifr.res.in
}

ABSTRACT: We study the effective dynamics of black hole horizons in Einstein-Maxwell theory in a large number of spacetime dimensions $D$. We demonstrate that horizon dynamics may be recast as a well posed initial value problem for the motion of a codimension one non gravitational membrane moving in flat space. The dynamical degrees of freedom of this membrane are its shape, charge density and a divergence free velocity field. We determine the equations that govern membrane dynamics at leading order in the large $D$ expansion. Our derivation of the membrane equations assumes that the solution preserves an $\mathrm{SO}(D-p-2)$ isometry with $p$ held fixed as $D$ is taken to infinity. However we are able to cast our final membrane equations into a completely geometric form that makes no reference to this symmetry algebra.

Keywords: Black Holes, Classical Theories of Gravity

ARXIV EPRINT: 1511.03432 


\section{Contents}

1 Introduction 1

2 The collective coordinate ansatz $\quad 6$

2.1 Boosted charged black holes in Kerr-Schild coordinates 6

$\begin{array}{lll}2.2 & \text { Collective coordinate spacetimes from boosted black holes } & 7\end{array}$

2.3 Subsidiary constraints on $\rho, u$ and $Q \quad 9$

$\begin{array}{lll}2.4 & \text { Fixing coordinate and gauge invariance } & 10\end{array}$

$\begin{array}{ll}2.5 \text { Perturbation theory } & 11\end{array}$

3 Perturbation theory assuming $\mathrm{SO}(D-p-2)$ invariance 11

3.1 Careful definition of the large $D$ limit 11

3.2 The Einstein-Maxwell equations in the $\mathrm{SO}(D-p-2)$ invariant sector $\quad 12$

$\begin{array}{lll}3.3 & \text { Setting up the perturbative computation } & 13\end{array}$

$\begin{array}{lll}\text { 3.3.1 Convenient coordinates for flat space } & 13\end{array}$

3.3.2 The perturbative expansion of $\mathrm{SO}(D-p-2)$ invariant solutions 13

3.3.3 More detailed parameterization of the first order corrections to the metric and gauge field 13

$\begin{array}{lll}\text { 3.3.4 Auxiliary embedding space } & 14\end{array}$

3.3.5 Constraints and Subsidiary conditions recast in auxiliary space $\quad 15$

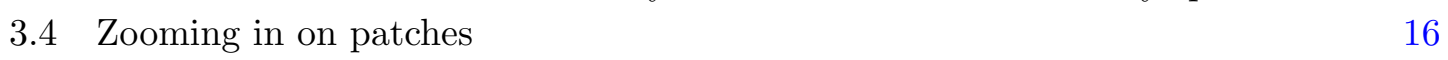

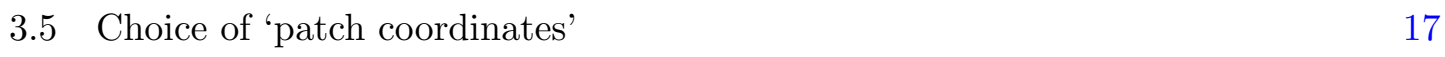

$\begin{array}{lll}3.6 & \text { The perturbative metric in a patch } & 18\end{array}$

$\begin{array}{lll}3.7 & \text { The structure of perturbative equations at first order } & 19\end{array}$

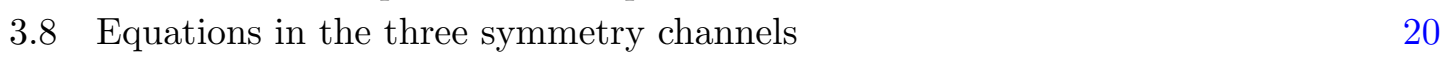

$\begin{array}{ll}3.9 \text { Basis for source functions } & 21\end{array}$

3.10 Equations of motion from regularity at the horizon 22

3.11 Equivalence to the equations of [1] in the uncharged limit 24

3.12 Conditions to fix the integration constants 25

3.13 Results for the first order correction on the patch 26

$\begin{array}{ll}\text { 3.13.1 The functions appearing in the gauge field } & 27\end{array}$

$\begin{array}{ll}\text { 3.13.2 The functions appearing in the metric } & 27\end{array}$

3.13.3 Correction (2nd order in $\frac{1}{D}$ ) to the scalar field $\phi \quad 28$

3.13.4 The $Q \rightarrow 0$ limit 28

3.14 The global first order metric $\quad 28$

4 Geometrical form of the first order corrected metric $\quad 29$

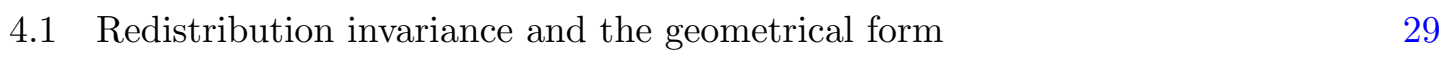

$\begin{array}{lll}4.2 & \text { Metric and gauge field in geometric form } & 30\end{array}$

4.2.1 The limit $Q \rightarrow 0 \quad 31$

4.3 Geometrizable form of the membrane equations of motion 32

4.3.1 Constraint equations and the membrane equations of motion 32

4.4 Comparison with the Reissner-Nordstrom solution 33 
5 Light quasinormal spectrum of the RN black hole $\quad 34$

$\begin{array}{ll}5.1 \text { The spectrum of shape fluctuations } & 37\end{array}$

$\begin{array}{lll}5.2 & \text { The spectrum of velocity fluctuations } & 39\end{array}$

$\begin{array}{ll}5.3 \text { The spectrum of charge fluctuations } & 40\end{array}$

5.4 A consistency check for shape fluctuations 41

6 Discussion 41

A Reissner-Nordstrom solution in Kerr-Schild coordinates 43

B Relating the geometric form of the metric and gauge field with the answer found in explicit computation $\quad 44$

B.1 Relating $\delta \phi$ to the geometric forms 45

$\begin{array}{lll}\text { C Relating equations of motion expressed in different forms } & 47\end{array}$

$\begin{array}{ll}\text { D Notation and translation } & 48\end{array}$

E Eigenvalues of the Laplacian for vector spherical harmonics 49

\section{Introduction}

Emparan, Suzuki, Tanabe (EST) and collaborators have recently noted [2-8] that the classical dynamics of black holes simplifies at large $D(D$ is the dimensionality of space time). Schwarzschild black holes in a large number of dimensions are characterized by two widely separated length scales. The first of these is the Schwarzschild radius $r_{0}$, while the second is the distance $\delta r$ away from Schwarzschild radius after which spacetime ceases to be warped by the black hole. In other words $\delta r$ is defined so that spacetime is effectively flat for $r>r_{0}+\delta r$. At large $D$ the membrane thickness, $\delta r$, is easily estimated; it turns out that $\delta r \sim r_{0} / D \ll r_{0}$. Similar observations apply to static charged black holes at large $D$.

The separation of scales between the membrane thickness and the black hole radius results in the simplification of black hole dynamics at large $D$. The first hint of this fact appeared in the results for the large $D$ spectrum of quasinormal modes of Schwarzschild black holes obtained by EST and collaborators $[5,7,8]$. It turns out that most of the quasinormal modes are heavy with frequencies $\sim 1 / \delta r$. The remaining modes are anomalously light; their frequencies are of order $1 / r_{0} \cdot{ }^{1}$ As we will see below, the spectrum of quasinormal modes about Reissner-Nordstrom black holes is qualitatively similar.

The pattern of the quasinormal mode frequencies described above may be understood intuitively as follows. A quasinormal mode is a linearized solution of Einstein's equations about the black hole background, subject to the condition that it is ingoing at the horizon and outgoing in the asymptotically flat exterior region. As the second boundary condition is effectively imposed at the outer edge of the membrane region, the quasinormal problem

\footnotetext{
${ }^{1}$ More precisely, all but a finite number of quasinormal modes at every angular momentum are heavy. A finite number of modes at every angular momentum are light.
} 
is analogous to the analysis of the harmonics of the wave equation in a hollow, leaky spherical shell. The radius of this shell is $r_{0}$ and its thickness is $\delta r$. Clearly modes with nonzero 'harmonic number' in the radial direction all have frequencies of order $1 / \delta r$; these are EST's generic heavy modes. Modes of zero radial harmonic number, if present, have frequencies of order $1 / r_{0}$; these are EST's anomalously light modes.

The imaginary part of all heavy quasinormal mode frequencies are of order $1 / \delta r$; it follows that these modes all decay away after a time scale of order $\delta r$. On the other hand the light quasinormal modes have lifetimes of order $r_{0}$. Consider a violent dynamical process like a black hole collision. For a time of order $\delta r$ after the event, dynamics is complicated and involves all quasinormal modes. For times $t \gg \delta r$, however, the heavy quasinormal modes have all decayed away and the subsequent dynamics is governed by a nonlinear interacting theory of only the light quasinormal modes, the principal focus of this paper. ${ }^{2}$

Light quasinormal modes may roughly be thought of as 'Goldstone bosons' for the symmetries of flat space that are broken by the black hole. Non rotating black holes appear in family of solutions labeled by a set of parameters $\alpha^{i}$; the black hole location, radius, boost velocity and charge. By infinitesimally varying each of these parameters we obtain a set of time independent linearized solutions of the Einstein-Maxwell equations about any of these black holes. Now consider configurations that locally resemble these zero modes but with $\delta \alpha^{i}=\delta \alpha^{i}(\theta)$, i.e. with the infinitesimal parametric variations chosen to be functions of the black hole angular coordinates with spherical harmonic numbers of order unity. It follows that in any patch of size of order $\delta r$ (i.e. of angular extent of order $\left.\delta r / r_{0}\right)$ the $\delta \alpha^{i}$ are approximately constant. In any such patch the fluctuation closely approximates a zero mode, and so is static on the time scale $\delta r$. However the variation of $\delta \alpha^{i}$ on length scales of order $r_{0}$ cause such configurations evolve over times of the same order. It follows that quasinormal modes built out of such configurations have frequencies of order $1 / r_{0}$, and may be identified with charged generalizations of the light modes of EST.

The identification of light quasinormal modes with 'Goldstone bosons' immediately suggests the possibility of using the collective coordinate method to derive the nonlinear 'chiral Lagrangian' of these light modes. ${ }^{3}$ On general grounds one expects that the effective nonlinear equations of motion for the light modes will admit a power series expansion in the ratio of the energy scales of the light and heavy modes, i.e. in a power series in $\delta r / r_{0} \sim 1 / D$. In other words the collective coordinate equations for light modes dynamics are a reformulation of black hole dynamics that is exact at large $D$.

At leading nontrivial order in $1 / D$, the equations that govern the collective coordinate dynamics of uncharged black holes were derived in the recent paper [1] (see [9-13] for closely related work). ${ }^{4}$ In this paper we build on the work of [1] in two different ways. First we

\footnotetext{
${ }^{2}$ At time scales large compared to $r_{0}$ the light quasinormal modes also decay away and the black holes settle down into their equilibrium state. The approach to equilibrium is governed by the linearized theory of quasinormal modes.

${ }^{3}$ As the resulting system turns out to be dissipative, however, it is easier to deal with the effective equations of motion than an effective action.

${ }^{4}$ The papers [9] and [10] worked out the effective collective coordinate expansions for the special case
} 
improve the construction of [1] in several respects. We use collective coordinate variables with a direct physical significance and present our final equations and spacetimes in an explicitly 'geometrical' form. Second - using the same improvements — we generalize the work of [1] to obtain the nonlinear collective coordinate dynamics of charged black holes in a large number of dimensions.

In the rest of this introduction we will provide a more detailed description of the collective coordinate construction presented in this paper and present our main results.

A more detailed introduction and summary. In the technical heart of this paper we follow [1] to simply write down a class of leading order collective coordinate spacetimes (see (2.5) below). We then carefully verify that our spacetimes and gauge fields (written down by physically guided guesswork following [1]) obey the Einstein-Maxwell equations of motion at leading order in $1 / D,{ }^{5}$ and so constitute a good starting point for the construction of true solutions to the Einstein-Maxwell equations in an expansion in $1 / D$. Our collective coordinate spacetimes are built sewing together patches of Reissner-Nordstrom black holes with different radii, charges and boost velocities into a single smooth spacetime. These spacetimes are in one to one correspondence with the configurations of a non gravitational codimension one membrane propagating in flat $D$ dimensional space. The dynamical degrees of freedom of the membrane are

1. The embedding of its timelike world volume in flat $D$ dimensional spacetime, i.e. the shape of the membrane. Through this paper we use the symbols $n_{A}$ and $K_{A B}$ to denote the normal and extrinsic curvature of the membrane surface in $D$ dimensional Minkowski space. We also use the symbol $\mathcal{K}=\eta^{A B} K_{A B}$ to denote the trace of the extrinsic curvature.

2. A velocity vector field $u^{A}$ in the membrane world volume (so that $u \cdot n=0$ ) whose world volume divergence vanishes (i.e. $\nabla \cdot u=0$ where $\nabla$ is the covariant derivative on the membrane world volume). The velocity field is normalized in the usual manner $u \cdot u=-1$.

3. A scalar charge density field $Q,{ }^{6}$ that lives on the membrane (this field is absent in the neutral case).

To reiterate, the starting point of the technical analysis presented in this paper is a class of 'collective coordinate spacetimes' - that are simply guessed. We have one such

of uncharged stationary configurations. When restricted to flat space and lowest order in $D$ the results of these papers are special cases of [1] and the current paper. The papers [11-13] analyze dynamics at length and time scales of order $r_{0} / \sqrt{D}$ (this turns out to be the relevant length scale for the Gregory-Laflamme phenomenon at large $D$ ), as opposed to the current paper where we focus on length scales of order unity.

${ }^{5}$ More precisely the equations of motion are obeyed everywhere outside the even horizons of these configurations. This is sufficient, as regions inside the event horizon are causally disconnected - and invisible - from those outside, and so may be ignored for the purposes of predicting observations outside the event horizon.

${ }^{6}$ More precisely the field $Q$ utilized in this paper is a variable proportional to the actual conserved charge density field on the membrane; see the upcoming paper [14] for details. 
spacetime for every distinct membrane configuration. Our collective coordinate spacetimes turn out to solve the Einstein-Maxwell equations at leading order in $1 / D$ everywhere outside their event horizons.

The strategy adopted in the rest of this paper is to use these spacetimes as the first term in the perturbative construction of true solutions of the Einstein-Maxwell equations in a power series expansion in $1 / D$. In this paper we explicitly implement this expansion to first subleading order in $1 / D$. In other words we correct the leading order collective coordinate spacetimes described above to ensure that they obey the Einstein-Maxwell equations not just at the leading order in $1 / D$ but also at first subleading order in this expansion. We discover that it is possible to accomplish this task with only nonsingular corrections if and only if the membrane shape, charge density and velocity fields obey the following local equations of motion 7,8

$$
\begin{aligned}
& \left(\frac{\nabla^{2} u}{\mathcal{K}}-\left(1-Q^{2}\right) \frac{\nabla \mathcal{K}}{\mathcal{K}}+u \cdot K-\left(1+Q^{2}\right)(u \cdot \nabla) u\right) \cdot \mathcal{P}=0, \\
& \frac{\nabla^{2} Q}{\mathcal{K}}-u \cdot \nabla Q-Q\left(\frac{u \cdot \nabla \mathcal{K}}{\mathcal{K}}-u \cdot K \cdot u\right)=0
\end{aligned}
$$

where $\nabla=$ the covariant derivative on the membrane world volume,

and $\mathcal{P}_{A B}=\eta_{A B}-n_{A} n_{B}+u_{A} u_{B}$

Corresponding to every solution of the equations (1.1) we are able to improve (2.5). The improvements are computed to ensure that the corrected configurations (see (4.1), (4.2), (4.3), (4.4), (4.5)) solve the Einstein-Maxwell equations at leading and first subleading order in $1 / D$. We expect the construction presented in this paper to constitute the first couple of terms in a systematic expansion of solutions to the Einstein-Maxwell equations order by order in $1 / D$.

As we have explained above, membrane spacetimes are parameterized by the shape of the membrane (one function), the charge density field (one function) and a unit normalized divergence free velocity field on the membrane ( $D-3$ functions) and so by $D-1$ functions in total. The membrane equations (1.1) are also $D-1$ in number (the first equation in (1.1) is a vector projected orthogonal to $n$ and $u$ and so has $D-2$ components, while the second is a scalar and so has one component). It follows that we have as many equations as variables and so (1.1) define an initial value problem for membrane motion. Eq. (1.1) are simply the large $D$ collective coordinate equations of black hole motion.

Following [1], in this paper we have derived the membrane equations (1.1) under the assumption that our spacetimes preserve an $\mathrm{SO}(D-p-2)$ isometry subgroup for $p$ held fixed

\footnotetext{
${ }^{7}$ The expression in the first bracket in the first of (1.1) is a vector in the membrane world volume and so is orthogonal to $n$. When acting on such a vector the projector $\mathcal{P}_{A B}=g_{A B}^{(W V)}+u_{A} u_{B}$ where $g_{A B}^{(W V)}$ is the induced metric on the membrane world volume.

${ }^{8}$ In the uncharged limit, the equation (1.1) are easily demonstrated to reduce to the membrane equation of motion presented in [1] once we account for the fact that the velocity field of this paper differs from the velocity field employed in [1] (see subsection 3.11 for relevant details.).
} 
as $D \rightarrow \infty .{ }^{9}$ Even though have made this assumption in our derivation, the final membrane equations (1.1) (and the spacetimes dual to solutions of these membrane equations) make no explicit reference to the isometry group. Our final equations are entirely covariant; they treat the isometry directions and other directions democratically. We refer to equations with this property as geometrical.

Given the geometrical nature of our membrane equations and spacetimes, it is natural to wonder whether our equations apply more generally than their derivation. Could it be that (1.1) captures the dynamics of black hole motions on time scales of order unity, even in the absence of a large isometry symmetry? While an appropriate version of such a conjecture might well be true, we would like to emphasize a subtlety. There are several pairs of independent geometrical expressions that reduce to each other at leading order in the large $D$ limit under the assumption of an $\mathrm{SO}(D-p-2)$ isometry but differ from each other more generally. ${ }^{10}$ For this reason it turns out that there are different geometrical ways of presenting the equations of motion (1.1), all of which are identical at leading order in $1 / D$ when evaluated on any membrane configuration that preserves an $\operatorname{SO}(D-p-2)$ isometry but which differ on more general configurations. As the results of this paper are all obtained assuming an $\mathrm{SO}(D-p-2)$ isometry, they cannot distinguish between these different geometrical presentations of the membrane equations. For example, the divergence of the first equation in (1.1) turns out to coincide, at leading order in large $D$, with the equation

$$
\left(1-Q^{2}\right)\left[\frac{\nabla^{2} \mathcal{K}}{\mathcal{K}^{2}}-\frac{u \cdot \nabla \mathcal{K}}{\mathcal{K}}\right]-\left(1+Q^{2}\right)\left(\frac{u \cdot \nabla \mathcal{K}}{\mathcal{K}}-u \cdot K \cdot u\right)=0
$$

where $\nabla=$ the covariant derivative on the membrane world volume,

under the assumption of $\mathrm{SO}(D-p-2)$ symmetry. It follows that the computations presented in this paper cannot resolve the question of which of these is the 'correct' leading order membrane equation in the absence of an isometry. ${ }^{11}$

The membrane equations (1.1) are nonlinear and rather complicated. In an upcoming paper [14] we demonstrate that these equations admit simple classes of solutions in which the membrane velocity field $u^{\mu}$ is that of rigid rotations and the charge density field is proportional to $u^{0}$ (the time component of the velocity vector). The membrane shape is constrained to obey a single nonlinear partial differential equation. Solutions obtained in this manner include the duals to charged rotating black hole solutions at large $D$. For the special case of uncharged black holes this nonlinear partial differential equation turns out to exactly match the constraint on the shape of stationary membranes derived in a

\footnotetext{
${ }^{9}$ This requirement guarantees that there are no unaccounted for factors of $D$ in, for instance, derivatives of the metric and gauge field.

${ }^{10}$ For example, the independent geometrical quantities $u \cdot \nabla \mathcal{K} / D$ and $\nabla_{\mu}(u \cdot K)^{\mu}$ may be shown to agree with each other at leading order in $1 / D$ for any membrane configuration that preserves an $\operatorname{SO}(D-p-2)$ invariance. On the other hand the same two expressions could differ at leading order when evaluated on configurations that do not enjoy any symmetry.

${ }^{11}$ Even staying within the class of isometric spacetimes, the iteration of the computations of this paper to one higher order could help to resolve this question. We hope to report on the results of a higher order computation in the not too distant future.
} 
different way in $[9,10]$, establishing that the results of $[9,10]$ (at leading order and in flat space) are a special case of the more general results of [1] and the current paper.

The simplest solution of the sort described in the previous paragraph is obtained upon switching off all angular velocities; the membrane solution is a static spherical 'soap bubble' with a uniform charge density. In section 4.4 below we have verified that the metric and gauge field dual to this solution agree perfectly with the exactly known static ReissnerNordstrom black hole solution expanded to first subleading order in $1 / D$ (we repeat this check for the much more nontrivial case of rotating black holes in the upcoming paper [14]).

The membrane equations (1.1) capture all of the complexities of black hole horizon dynamics at large $D$, at time scales of order unity. ${ }^{12}$ The detailed study of (1.1) should teach us a great deal about black hole horizon dynamics. As a first small step in this program, in section (5) we linearize the membrane equations (1.1) about the exact spherical solution dual to the Reissner-Nordstrom black hole, and determine the spectrum of small fluctuations about this background (see section 4.4) for details. This spectrum of linearized fluctuations may be regarded as a prediction for the spectrum of light quasinormal modes about charged black holes at large $D$.

In the course of obtaining the quasinormal mode spectrum described in the previous paragraph, we reduce the manifestly geometrical but slightly abstract equations (1.1) to explicit linear differential equations for two scalar fields and a divergence free vector field on $S^{D-2}$ times time (this reduction is valid for linearized fluctuations about the spherical membrane surface). This explicit form of the equations helps us verify that the equations (1.1) do indeed constitute a well posed initial value problem for the membrane shape, charge density and velocity fields at least for these linearized configurations, as we had anticipated above on intuitive grounds. Our explicit results for the quasinormal modes also reveals that the membrane equations (1.1) are highly dissipative. As an independent test of the equations (1.1) it would be useful to verify our prediction for the large $D$ quasinormal spectrum by direct analysis of the Einstein-Maxwell equations about the Reissner-Nordstrom black hole background. While we make some remarks about this, we leave a detailed verification to future work.

\section{The collective coordinate ansatz}

\subsection{Boosted charged black holes in Kerr-Schild coordinates}

The Reissner-Nordstrom black hole in the 'Kerr-Schild' coordinate system ${ }^{13}$ is given by

$$
\begin{aligned}
d s^{2} & =-d t^{2}+d r^{2}+r^{2} d \Omega_{D-2}^{2}+\left(\left(1+Q^{2} c_{D}\right)\left(\frac{r_{0}}{r}\right)^{D-3}-c_{D} Q^{2}\left(\frac{r_{0}}{r}\right)^{2(D-3)}\right)(d t+d r)^{2}, \\
& =d s_{\text {flat }}^{2}+\left(\left(1+Q^{2} c_{D}\right)\left(\frac{r_{0}}{r}\right)^{D-3}-c_{D} Q^{2}\left(\frac{r_{0}}{r}\right)^{2(D-3)}\right)(d t+d r)^{2} \\
A & =\sqrt{2} Q\left(\frac{r_{0}}{r}\right)^{D-3}(d t+d r) .
\end{aligned}
$$

\footnotetext{
${ }^{12}$ We believe this to be true at least for spacetimes that preserve an $\mathrm{SO}(D-p-2)$ isometry for any $p$ that is held fixed as $D$ is taken to infinity.

${ }^{13}$ See appendix A for a lightning introduction to this coordinate system and its advantages.
} 
Eq. (2.1) describes a black hole at rest, i.e. a black hole moving with velocity $u=-d t$. The solution for a black hole moving at an arbitrary constant velocity $u$ may be obtained by boosting (2.1) and is given by

$$
\begin{array}{rlrl}
g_{M N} & =\eta_{M N}+\left(\left(1+Q^{2} c_{D}\right) \frac{1}{\rho^{D-3}}-c_{D} Q^{2} \frac{1}{\rho^{2(D-3)}}\right) O_{M} O_{N}, & & \\
A_{M} & =\frac{\sqrt{2} Q O_{M}}{\rho^{D-3}}, & & \\
O & =n-u, \quad u=\text { const }, \quad n=-1, \quad \rho=\frac{r}{r_{0}}, \\
r^{2} & =P_{M N} x^{M} x^{N}, \quad P_{M N}=\eta_{M N}+u_{M} u_{N}, \quad n=r_{0} d \rho, \quad & \text { note } u \cdot n=0 .
\end{array}
$$

Note that the function $\rho$ in (2.2) obeys the identity

$$
\rho \nabla^{2} \rho=(D-2) d \rho \cdot d \rho .
$$

Here and through most of this paper we view $\rho$ as a function that lives in flat $D$ dimensional space. In particular $\nabla$ in (2.3) is the covariant derivative in flat space rather than in the metric (2.2).

Through this paper we will use the term membrane to refer to the surface $\rho=1$ viewed as a submanifold of flat Minkowski space. Note also that $u^{\mu}$ may be thought of a vector field that lives on the membrane. It is obvious that

$$
\nabla \cdot u=0
$$

where $\nabla$ is the covariant derivative on the membrane.

\subsection{Collective coordinate spacetimes from boosted black holes}

Consider the spacetime given by

$$
\begin{aligned}
g_{M N} & =\eta_{M N}+\left[\left(1+Q^{2}\right) \frac{1}{\rho^{D-3}}-\frac{Q^{2}}{\rho^{2(D-3)}}\right] O_{M} O_{N}, \\
A_{M} & =\frac{\sqrt{2} Q O_{M}}{\rho^{D-3}}, \\
O & =n-u, \quad u \cdot u=-1, \quad n=\frac{d \rho}{\sqrt{d \rho \cdot d \rho}}, \quad u \cdot n=0,
\end{aligned}
$$

where $\rho, Q$ and $u$ are arbitrary smooth functions and vector fields in flat $D$ dimensional Minkowski spacetime subject only to the requirement that the function $\rho$ obeys (2.3) on the membrane surface and that the velocity field restricted to the membrane obeys (2.4).

The codimension one membrane worldvolume will play a special role in this paper. We assume that the function $\rho$ is chosen to ensure that the membrane surface is a smooth timelike submanifold of flat Minkowski space. ${ }^{14}$ The membrane separates regions of spacetime where with $\rho<1$ (inside the membrane) from regions with $\rho>1$ (outside the membrane).

\footnotetext{
${ }^{14}$ We will see below that the same surface $-\rho=1$ - is a null when viewed as a submanifold of the metric (2.5).
} 
The function $\rho$ is chosen to ensure that the outside region is a connected spacetime and that includes all of spacelike infinity as well as $\mathcal{I}^{+}$and $\mathcal{I}^{-}$. The membrane worldvolume itself is not necessarily connected.

The spacetimes (2.5) have the following properties.

1. Upto corrections of order $1 / D$, the static black holes (2.2) are special cases of (2.5) with the $\rho, Q$ and $u$ functions given as in (2.2). In these special cases $\rho=1$ is the black hole event horizon.

2. It is easily verified the membrane surface $\rho=1$ is a null submanifold of the metric (2.5) for a general spacetime of this form. At least when (2.5) settles down to a stationary black hole at late times (as we will assume throughout this paper) this submanifold may be identified with the spacetime event horizon. ${ }^{15}$

3. Consider a point $x_{0}^{\mu}$ on the membrane $(\rho=1)$ of the spacetime (2.5). Let $u_{0}^{\mu}, Q_{0}$ and $\mathcal{K}_{0}$ denote the velocity, charge density field and trace of membrane extrinsic curvature at that point. Comparing with (2.2), we will see in subsection 3.6 below that a patch of size of order $\frac{1}{D}$ centered about $x_{0}^{\mu}$ is identical, at leading order in $D$, to the metric and gauge field of a patch centered about the membrane of a Reissner-Nordstrom black hole of radius $(D-2) / \mathcal{K}, Q$ parameter $Q_{0}$ and boost velocity $u_{0}^{\mu}$.

4. It seems plausible from point (3) above that every patch centered about the membrane of the configuration (2.5) obeys the Einstein-Maxwell equations at leading order in $1 / D$. In subsection 3.6 below we demonstrate that this is the case provided the spacetime (2.5) enjoys an $\mathrm{SO}(D-p-2)$ isometry for any $p$ that is held fixed as $D$ is taken to infinity.

5. The gauge field in (2.5) and the deviation of the metric from $d s_{\text {flat }}^{2}$ scales like $e^{-D(\rho-1)}$. It follows (2.5) approaches flat space exponentially rapidly for $\rho-1 \gg 1 / D$.

6. Combining (4) and (5) above it follows that (2.5) also obeys the Einstein-Maxwell equations at leading order in $1 / D$ (or better) everywhere outside its event horizon.

7. The equations of motion are not well solved when $1-\rho \gg 1$. However points that lie inside the event horizon of (2.5) are causally disconnected from dynamics on and outside the membrane and will be ignored in the rest of this paper.

In summary, the metric (2.5) is built by stitching together bits of the event horizon of Reissner-Nordstrom black holes of varying radii, charge densities and boost velocities. The spacetime (2.5) obeys the Einstein-Maxwell equations at leading order in large $D$ everywhere outside its horizon at least provided it preserves an $\mathrm{SO}(D-p-2)$ isometry. It follows that metrics of the form (2.5) are useful starting points for a perturbative construction of the solutions of the Einstein-Maxwell equation in an expansion in $\frac{1}{D}$.

\footnotetext{
${ }^{15}$ The dissipative nature of the membrane equations of motion we derive below suggests that all solutions reduce to stationary solutions at late times.
} 


\subsection{Subsidiary constraints on $\rho, u$ and $Q$}

The spacetimes (2.5) are parameterized by the functions $\rho$ and $Q$ and $u^{\mu}$. These functions are defined on all of $D$ dimensional Minkowski space. However we have already noted that (2.5) rapidly tends to flat space when $\rho-1 \gg \frac{1}{D}$. Consequently two spacetimes whose $\rho$ and $Q$ and $u^{\mu}$ functions agree on the surface $\rho=1$ but deviate at larger values of $\rho$ actually describe spacetimes that agree at leading order in $1 / D$ on and outside their event horizons. ${ }^{16}$

In this paper we use spacetimes of the form (2.5) as the starting point for a perturbative expansion of true solutions of the Einstein-Maxwell system in a power series in $1 / D$. Any two configurations of the form (2.5) that differ from each other only at subleading orders in $1 / D$ constitute equivalent starting points for perturbation theory. In order to restrict attention only to inequivalent configurations it is convenient to invent a set of rules that determine the functions $\rho, u$ and $Q$ everywhere in spacetime, in terms of the shape of the membrane and the values of the velocity and charge density fields on the membrane. We refer to these arbitrary rules as subsidiary constraints on the functions $\rho, Q$ and $u$.

There is a great deal of freedom in the choice of subsidiary constraints. Two different choices of these conditions lead to the same solution at any given order in perturbation theory. The differences between the starting points in perturbation theory are compensated for by the differences in the results of the perturbative expansion.

While all choices of subsidiary constraints are on equal footing in principle, in practice some choices (those that most accurately approximate the true eventual solutions) lead to simpler results in perturbation theory than others. After experimenting with a few options we have chosen, in this paper, to impose the following subsidiary constraints on $\rho, u$ and $Q$ :

$$
\begin{aligned}
& \rho \nabla^{2} \rho=(D-2) d \rho \cdot d \rho, \\
& u \cdot u=-1, \quad n \cdot u=0, \quad \mathcal{P}^{M N}\left[(n \cdot \nabla) u_{M}+(u \cdot \nabla) n_{M}\right]=0, \\
& n \cdot \nabla Q=0,
\end{aligned}
$$

where $n=\frac{d \rho}{\sqrt{d \rho \cdot d \rho}}, \quad \mathcal{P}^{M N}=\eta^{M N}-n^{M} n^{N}+u^{M} u^{N}$.

and $\nabla=$ the covariant derivative in the embedding flat space.

Let us pause to comment on our choice of subsidiary constraints. Recall that it is an important element of our construction that (2.6) is obeyed on the surface $\rho=1$ (see (2.3)). This is a physical requirement, independent of arbitrary choices of subsidiary conditions. Our first subsidiary condition (2.6) simply asserts that (2.3) continues hold everywhere;

\footnotetext{
${ }^{16}$ In and around subsection 3.6 we show that for this statement to be true it is also necessary the gradients $\nabla \rho$ of the two $\rho$ functions coincide on the membrane $\rho=1$ at leading order in the large $D$ limit. However this is automatic, given the conditions we have imposed on our construction. Upto a position dependent normalization, $\nabla \rho$ is proportional to the normal vector of the surface $\rho=1$. It follows that the two $\nabla \rho$ functions agree with each other upto normalization at $\rho=1$. The condition that both $\rho$ functions obey (2.3) at $\rho=1$ guarantees that the normalizations also agree at leading order in the large $D$ limit (see (3.16)).
} 
even away from the membrane. This condition is sufficient to determine the function everywhere in terms of the shape of the membrane (i.e. solutions to the equation $\rho-1=0$ ).

The third condition in (2.6) asserts that $Q$ is defined off the membrane surface by parallel transporting it along integral curves of the normal vector $n \propto d \rho$. The second condition (2.6) determines $u$ in terms of its value on the membrane by specifying its evolution under parallel transport under the same integral curves. ${ }^{17}$

\subsection{Fixing coordinate and gauge invariance}

In the next section we will describe the perturbative procedure we will employ to correct the spacetime (2.5) in order to obtain a spacetime that solves the Einstein-Maxwell equations upto first subleading order in $1 / D$. In order to find an unambiguous solution to this problem we need to fix coordinate redefinition and Maxwell gauge ambiguities. In this subsection we describe our choice of coordinates and gauge.

Let the spacetime metric in the solutions described by this paper take the form

$$
g_{M N}=\eta_{M N}+h_{M N},
$$

where $h_{M N}$ is given, at leading order, by (2.5). We fix coordinate redefinition ambiguity by imposing the condition

$$
O^{M} h_{M N}=0,
$$

where

$$
O=n-u,
$$

and all indices in (2.8) are raised and lowered using the flat metric $\eta_{M N}$. Using the fact that $O \cdot O=0$, it is easily verified that the leading order metric (2.5) does indeed obey (2.8).

In a similar manner we fix the Maxwell gauge ambiguity by imposing the condition

$$
O^{M} A_{M}=0 .
$$

Note that (2.10) is obeyed at leading order (see (2.5)).

Note that our choice of gauge depends on $O$, and so on $n$ and $u$, which, in turn, depend on the membrane shape and velocity field in the particular solution under study. Our choice of gauge is somewhat analogous to a background field gauge in the study of gauge theories, or, more closely, to the gauges adopted in the study of the fluid gravity correspondence (see e.g. [15-20]).

Note also that the coordinate choice adopted in this paper differs in detail from that of [1]. As is clear from the discussion of this section, the gauge adopted here is completely geometrical. This is not true of the gauge adopted in [1], which singles out the isometry direction as special.

\footnotetext{
${ }^{17}$ The subsidiary constraints adopted in this paper are chosen to permit simple comparison with exact uncharged rotating black hole solutions, see [14] for details. These conditions imposed in this differ from the rather elegant geometrical subsidiary constraints imposed in [1].
} 


\subsection{Perturbation theory}

In the next section we will implement a perturbative procedure that can be used to correct $(2.5)$ at first subleading order in $1 / D$. Roughly speaking we search for a metric and gauge field of the form

$$
\begin{aligned}
g_{M N} & =\eta_{M N}+h_{M N}, \\
h_{M N} & =\sum_{n=0}^{\infty} \frac{h_{M N}^{(n)}}{D^{n}}, \\
A_{M} & =\sum_{n=0}^{\infty} \frac{A_{M}^{(n)}}{D^{n}}, \\
h_{M N}^{(0)} & =O_{M} O_{N}\left[\left(1+Q^{2}\right) \rho^{D-3}-Q^{2} \rho^{-2(D-3)}\right], \\
A_{M}^{(0)} & =\frac{\sqrt{2} Q}{\rho^{D-3}},
\end{aligned}
$$

and attempt to find the correction fields $h_{M N}^{(1)}$ and $A_{M}^{(1)}$ that ensure that the EinsteinMaxwell equations are satisfied not just at leading order but also at first subleading order in $1 / D$. In order to technically implement this idea, it turns out to be very helpful to assume our solutions preserve a large isometry group, as we describe in detail in the next section.

\section{Perturbation theory assuming $\mathrm{SO}(D-p-2)$ invariance}

\subsection{Careful definition of the large $D$ limit}

In the computational part of this paper we follow [1] to take the limit $D \rightarrow \infty$ while preserving an $\mathrm{SO}(D-p-2)$ symmetry with $p$ held fixed. We take the large $D$ limit while maintaining a large isometry subgroup so that we can reliably estimate the scaling with $D$ of all terms in the equations we encounter.

The requirement that our solutions preserve an isometry group is less restrictive than it first appears for two reasons. First, several spacetimes of physical interest (e.g. those that describe classes of black hole collisions) indeed preserve large isometry groups. Secondly, although the derivation of the membrane equations that we present below assumes an $\mathrm{SO}(D-p-2)$ isometry, we will see that all our final equations are entirely geometrical on the membrane world volume; the isometry directions are not special in any way. In particular our final equations are independent of $p$.

While none of our final results will depend on $p$, all intermediate computations are performed within a framework that explicitly preserves $\mathrm{SO}(D-p-2)$ invariance. In order to perform computations we assume that our metric and gauge field take the form

$$
\begin{aligned}
d s^{2} & =g_{\mu \nu}\left(x^{\mu}\right) d x^{\mu} d x^{\nu}+e^{\phi\left(x^{\mu}\right)} d \Omega_{d}^{2}, \\
A & =A_{\mu}\left(x^{\mu}\right) d x^{\mu}, \\
d & =D-p-3, \quad \mu=1 \ldots p+3,
\end{aligned}
$$


where $g_{\mu \nu}, \phi$ and $A_{\mu}$ are all arbitrary functions of the coordinates $x^{\mu}$ but are independent of the angular coordinates on the $S^{d}$ in (3.1). ${ }^{18}$ Under this assumption the $D$ dimensional Einstein-Maxwell equations effectively reduce to a $p+3$ dimensional Einstein-Maxwell system coupled to the effective scalar field $\phi$.

\subsection{The Einstein-Maxwell equations in the $\mathrm{SO}(D-p-2)$ invariant sector}

In this paper we study solutions of the Einstein-Maxwell equations governed by the Lagrangian

$$
\mathcal{S}=\frac{1}{16 \pi G_{D}} \int \sqrt{-\tilde{g}} d^{D} x\left(\tilde{R}-\frac{F_{M N} F^{M N}}{4}\right)
$$

where $^{19}$

$$
\begin{aligned}
F_{M N} & =\partial_{M} A_{N}-\partial_{N} A_{M} \\
\tilde{R} & =\text { Ricci scalar in full D dimensional spacetime, } \\
\tilde{g} & =\text { Determinant of the metric in full D dimensional spacetime. }
\end{aligned}
$$

We wish to focus attention on metrics and gauge fields of the form (3.1). In this section we will work out the effective dynamical equations for such configurations.

Substituting (3.1) into (3.3) we find the effective Lagrangian ${ }^{20}$

$$
\begin{aligned}
\mathcal{S} & =\frac{\Omega_{d}}{16 \pi G_{D}} \int \sqrt{-g} d^{p+3} x e^{\frac{d \phi}{2}}\left(R+d(d-1) e^{-\phi}+\frac{d(d-1)}{4}(\partial \phi)^{2}-\frac{F_{\mu \nu} F^{\mu \nu}}{4}\right), \\
(\partial \phi)^{2} & =g^{\mu \nu}\left(\partial_{\mu} \phi\right)\left(\partial_{\nu} \phi\right) .
\end{aligned}
$$

Varying this Lagrangian we obtain the equations of motion

$$
\begin{aligned}
& (d-1) e^{-\phi}-\frac{d}{4}(\partial \phi)^{2}-\frac{1}{2} \nabla^{2} \phi+\frac{1}{4(d+p+1)} F_{\mu \nu} F^{\mu \nu}=0 \\
& R_{\mu \nu}-\frac{d}{4}\left(\partial_{\mu} \phi\right)\left(\partial_{\nu} \phi\right)-\frac{d}{2} \nabla_{\mu} \nabla_{\nu} \phi-\frac{1}{2} F_{\mu \rho} F_{\nu}{ }^{\rho}+\frac{1}{4(d+p+1)} F_{\rho \sigma} F^{\rho \sigma} g_{\mu \nu}=0 \\
& \nabla_{\mu} F^{\mu \nu}+\frac{d}{2}\left(\partial_{\mu} \phi\right) F^{\mu \nu}=0
\end{aligned}
$$

where $d=D-p-3$,

and $\nabla=$ covariant derivative taken w.r.t. the metric $g_{\mu \nu}$.

\footnotetext{
${ }^{18}$ In the special case of flat space

$$
d s^{2}=\eta_{\alpha \beta} d w^{\alpha} d w^{\beta}+d S^{2}+S^{2} d \Omega_{d}^{2}=\eta_{\alpha \beta} d w^{\alpha} d w^{\beta}+d z_{M} d z^{M},
$$

where $z^{M}$ are the $d+1$ Euclidean coordinates built out of angular coordinates on $S^{d}$ and the radial coordinate $S$.

${ }^{19}$ In (3.4) the gauge field $A_{\mu}$ and the metric $g_{\mu \nu}$ are both taken to be dimensionless while Newton's constant $G_{D}$ has length dimension $D-2$.

${ }^{20}$ Due to the presence of $\mathrm{SO}(D-p-2)$ symmetry all the quantities depend only on $w^{\alpha}, S$ coordinates, while all the vectors (in particular, $A$ ) have components only in $d w^{\alpha}, d S$ directions. Hence when we go to the $p+3$ dimensional space, the $M, N$ indices are replaced by $\mu, \nu$.
} 


\subsection{Setting up the perturbative computation}

\subsubsection{Convenient coordinates for flat space}

The metric (2.5) is completely determined once we specify the two scalar fields $\rho$ and $Q$ and the vector field $u^{\mu}$. These fields live in flat space and are constrained to obey the equation (2.6).

The following coordinates for flat space

$$
d s_{\text {flat }}^{2}=\eta_{\alpha \beta} d w^{\alpha} d w^{\beta}+d S^{2}+S^{2} d \Omega_{d}^{2}, \quad i=\{0,1, \cdots, p+1\}, \quad d=D-p-3 .
$$

are particularly useful for studying $\mathrm{SO}(D-p-2)$ invariant configurations. In these coordinates the requirement of $\mathrm{SO}(D-p-2)$ isometry implies that $\rho, Q$ and $u$ are functions of $\left(\left\{w^{\alpha}, S\right\} \equiv\left\{x^{\mu}\right\}\right)$ only. Moreover $u_{\theta_{i}}=0$ in every angular direction $\theta_{i}$ on the $S^{d}$.

\subsubsection{The perturbative expansion of $\operatorname{SO}(D-p-2)$ invariant solutions}

Metrics and gauge fields that preserve an $\mathrm{SO}(D-p-2)$ isometry can be parameterized in the form

$$
\begin{aligned}
d s^{2} & =g_{\mu \nu}\left(S, w^{\alpha}\right) d x^{\mu} d x^{\nu}+S^{2} e^{\delta \phi\left(S, w^{\alpha}\right)} d \Omega_{d}^{2}, \\
A_{M} d X^{M} & =A_{\mu}\left(S, w^{\alpha}\right) d x^{\mu} .
\end{aligned}
$$

Note that

$$
\phi=\phi^{0}+\delta \phi, \quad \phi^{0}=2 \ln (S) .
$$

( $\phi^{0}$ is simply value of $\phi$ in flat space).

As explained around (2.7), in this paper we will expand the metric and gauge field in a power series expansion in $1 / D .^{21}$ The schematic expansion (2.7) takes the precise form

$$
g_{\mu \nu}=\sum_{k=0}^{\infty}\left(\frac{1}{D}\right)^{k} g_{\mu \nu}^{(k)}, \quad A_{\mu}=\sum_{k=0}^{\infty}\left(\frac{1}{D}\right)^{k} A_{\mu}^{(k)}, \quad \delta \phi=\sum_{k=1}^{\infty}\left(\frac{1}{D}\right)^{k} \delta \phi^{(k)} .
$$

From (2.5) we read off the leading values of $g_{\mu \nu}$ and $A_{\mu}$

$$
\begin{aligned}
g_{\mu \nu}^{(0)} d x^{\mu} d x^{\nu} & =\eta_{\alpha \beta} d w^{\alpha} d w^{\beta}+d S^{2}+\left[\left(1+Q^{2}\right) \rho^{-(D-3}-Q^{2} \rho^{-2(D-3)}\right]\left(O_{\mu} d x^{\mu}\right)^{2}, \\
A_{\mu}^{(0)} & =\sqrt{2} Q \rho^{-(D-3)} O_{\mu} .
\end{aligned}
$$

\subsubsection{More detailed parameterization of the first order corrections to the met- ric and gauge field}

After imposing the gauge conditions (2.8) and (2.10), the metric correction $g_{\mu \nu}^{(1)}$ and gauge field correction $A_{\mu}^{(1)}$ can can be parameterized in terms of 6 unknown scalar, three unknown vector and one unknown tensor functions ${ }^{22}$ as

$$
\begin{aligned}
g_{\mu \nu}^{(1)}= & S_{(V V)} O_{\mu} O_{\nu}+2 S_{(V z)} O_{(\mu} Z_{\nu)}+S_{(z z)} Z_{\mu} Z_{\nu}+S_{(T r)} P_{\mu \nu} \\
& +2 V^{(V)}{ }_{(\mu} O_{\nu)}+2 V^{(z)}{ }_{(\mu} Z_{\nu)}+T_{\mu \nu}, \\
A_{\mu}^{(1)}= & S_{(A V)} O_{\mu}+S_{(A z)} Z_{\mu}+V_{\mu}^{(A)},
\end{aligned}
$$

\footnotetext{
${ }^{21}$ The central advantage of the assumption of $\mathrm{SO}(D-p-2)$ isometry is that the variables of the perturbation expansion are independent of $D$.

${ }^{22}$ The terms scalar, vector and tensor refer to the transformation properties of the fields under those rotations in the tangent space that leave $n, u$ and $d S$ fixed. See below for more details.
} 
where

$$
\begin{aligned}
O & =n-u, \quad Z=\frac{d S}{S}-\left(\frac{n \cdot d S}{S}\right) n, \\
P_{\mu \nu} & =\text { projector perpendicular to } u, n \text { and } Z, \quad P^{\mu \nu} T_{\mu \nu}=0 .
\end{aligned}
$$

The vectors $\left(V_{\mu}^{(V)}, V_{\mu}^{(Z)}, V_{\mu}^{(A)}\right)$ and tensor $\left(T_{\mu \nu}\right)$ above are all projected orthogonal to $O$, $n$ and $Z$ (the tensor $T_{\mu \nu}$ is also assumed to be traceless).

Let us now consider the corrections of the 'dilaton' function $\delta \phi$. We see from (3.19) and (3.20) that $\chi=D(d \phi)$ appears in the equations of motion. Were $\phi$ to have an $\mathcal{O}\left(\frac{1}{D}\right)$ fluctuation $\delta \phi^{(1)}$, this term would contribute to the equations of motion at leading order, invalidating the fact that the starting metric (2.5) solves the Einstein-Maxwell equations at leading order. For the same reason $\phi$ at $\mathcal{O}\left(\frac{1}{D}\right)^{2}$, contributes to the Einstein-Maxwell equations at $\mathcal{O}\left(\frac{1}{D}\right)$. It follows that $\delta \phi^{(2)}$ is an unknown function that contributes to the first order perturbative equations at the same order as the 6 scalars that appear in (3.11), and so will have to be determined together with these six functions in the computation of the first corrections to (2.5).

\subsubsection{Auxiliary embedding space}

The coordinate system (3.7) describes flat $R^{D}$ as the 'fibration' of an $S^{d}$ over a $p+3$ dimensional base space with metric

$$
d s_{\text {flat }}^{2}=\eta_{\alpha \beta} d w^{\alpha} d w^{\beta}+d S^{2}=\eta_{\mu \nu} d x^{\mu} d x^{\nu}, \quad x^{\mu}=\left\{w^{\alpha}, S\right\} .
$$

The radius of the fibred $S^{d}$ is given by the coordinate $S$.

Under the assumption of $\mathrm{SO}(D-p-2)$ symmetry, the membrane world volume can be thought of as a codimension one ( $p+2$ dimensional) surface in the base space together with the $d$ dimensional spheres fibred over each of the base points on this surface. More generally all the ingredients - the functions $\rho, u^{\mu}$ and $Q$ - that go into the construction of the seed metric (2.5) can all be regarded as functions and vector fields on the base space - which then determine $\mathrm{SO}(d+1)$ invariant functions and vector fields on all of $R^{D}$ in the obvious manner. This is the viewpoint we will adopt while doing the computations described in this section. This viewpoint is convenient because the auxiliary space (3.12) makes no reference to $D$. Once we formulate our perturbation theory in terms of fields propagating on the auxiliary space (3.12), all factors of $D$ in the equations are completely manifest, allowing for a clean formulation of large $D$ perturbation theory.

The end result of the first stage of our computation (e.g. the results presented in (3.13)) are all presented in terms of covariant derivatives of the field $\phi, u$ and $Q$ viewed as scalar and one-form fields that live in the base or auxiliary space (3.12).

It is important to note general expressions built out of covariant derivatives of $\mathrm{SO}(D-$ $p-2)$ invariant fields in the auxiliary space (3.12) do not agree with the corresponding expressions built out of covariant derivatives of the same fields in the metric (3.7) of the 
embedding space. ${ }^{23}$ In appendix $\mathrm{D}$ we have explored the dictionary between covariant expressions in the full flat $D$ dimensional space and the auxiliary space. Using these translation formulae, we are then able to rewrite our final results for the first order corrected metric and gauge fields in terms of full spacetime covariant derivatives of $\rho, u$ and $Q$. Our final results, presented in the next section, are given in this language, and turn out to be geometrical, in a sense we describe in detail below.

\subsubsection{Constraints and Subsidiary conditions recast in auxiliary space}

As we have explained in the previous section our construction (2.5) works provided the functions $\rho$ and $u$ obey the conditions (2.3) and (2.4). The $\nabla^{2}$ in (2.3) is a Laplacian in the full flat space (3.7), while the $\nabla$ operator in (2.4) is the covariant derivative on the membrane, viewed as a submanifold of the full flat space (3.7). In order to use these conditions in our computations below, we need to rewrite them in terms of covariant derivatives on (3.12) and on the membrane world volume viewed as a submanifold of (3.12). ${ }^{24}$

Depending on context, we will use the symbol $\tilde{\nabla}$ to denote the covariant derivative either in the base space (3.12) or on the membrane viewed as a submanifold of (3.12). As we have explained in appendix D,

$$
\nabla \cdot u=(D-p-2) Z \cdot u+\tilde{\nabla} \cdot u
$$

(in this equation $\nabla$ is the covariant derivative of the membrane viewed as a submanifold of $R^{D}$ while $\tilde{\nabla}$ is the covariant derivative on the membrane viewed as a submanifold of $(3.12))$. Here

$$
Z=\frac{d S}{S}-\left(\frac{n \cdot d S}{S}\right) n
$$

Using the fact that (2.4) is assumed to hold for our ansatz metrics it follows from (3.14) that

$$
Z \cdot u=-\frac{\tilde{\nabla} \cdot u}{D-p-2}
$$

In a similar manner the fact that (2.3) is assumed to hold on the membrane of (2.5) implies that

$$
(D-p-2) \frac{d S \cdot \tilde{\nabla} \rho}{S}+\tilde{\nabla}^{2} \rho=(D-2) d \rho \cdot d \rho
$$

where $\tilde{\nabla}=$ the covariant derivative on the space $(5.9)$.

\footnotetext{
${ }^{23}$ Roughly speaking the difference comes about in terms involving expressions like $\Gamma_{S M}^{M}$ with $M$ summed over. This expression receives contributions from $M$ ranging over the angular directions of $\Omega_{d}$ in the case of (3.7) but not in the case of (3.12).

${ }^{24}$ All computations in the paper [1] were performed in the auxiliary space (3.12). The final results of [1] were presented in this auxiliary space, without being reconverted to the full space. Note also that in the auxiliary space, because of our choice of coordinates, all Christoffel symbols vanish and the covariant derivatives are same as partial derivatives.
} 
In an entirely analogous manner, the subsidiary condition (2.6) can be recast in terms of covariant derivatives in the auxiliary space (3.12).

$$
\begin{aligned}
& (D-p-2) \frac{\rho}{S} d S \cdot \tilde{\nabla} \rho+\rho \tilde{\nabla}^{2} \rho=(D-2) d \rho \cdot d \rho, \\
& u_{\mu} u^{\mu}=-1, \quad n_{\mu} u^{\mu}=0, \\
& \left(\eta^{\mu \nu}+u^{\mu} u^{\nu}-n^{\mu} n^{\nu}\right)\left[\left(n^{\alpha} \tilde{\nabla}_{\alpha}\right) u_{\mu}+\left(u^{\alpha} \tilde{\nabla}_{\alpha}\right) n_{\mu}\right]=0 \\
& n^{\mu} \tilde{\nabla}_{\mu} Q=0, \\
& \text { where } n_{\mu}=\frac{\tilde{\nabla}_{\mu} \rho}{\sqrt{\left(\tilde{\nabla}_{\nu} \rho\right)\left(\tilde{\nabla}^{\nu} \rho\right)}} \text {, } \\
& \text { and } \tilde{\nabla}=\text { the covariant derivative on (3.12). }
\end{aligned}
$$

\subsection{Zooming in on patches}

In this subsection we will identify a scaling limit of distance scales that admits an interesting large $D$ limit. For this purpose we turn back to the Einstein-Maxwell equations specialized to the case of $\operatorname{SO}(D-p-2)$ invariant configurations and note that derivatives of the scalar field $\phi$ appear in (3.6) with additional factors of $D$ as compared to terms with an equal number of derivatives of $g_{\mu \nu}$ or $A_{\mu}$. This observation (see [1]) suggests that we will obtain one class of nontrivial solutions to these equations if we assume that $g_{\mu \nu}$ and $A_{\mu}$ vary on length scale $1 / D$, i.e. the length scale of $\delta r$ (see the introduction) while $\phi$ varies at the length scale unity (at least upto corrections that are subleading in $1 / D$ ). Under this assumption the solutions we study are characterized by two widely separated length scales, exactly like the black holes described in the introduction. ${ }^{25}$

In order to describe the large $D$ limit of solutions characterized by two different length scales $(1 / D$ and unity) we adopt the following procedure. We view our manifold as a union of patches, each of size $1 / D$. Each patch is centered around a particular coordinate $x_{0}^{\mu}$. In each such patch we work with the scaled coordinates, metric, connections and gauge fields

$$
\begin{aligned}
x^{\mu} & =x_{0}^{\mu}+\frac{\alpha^{\mu}\left(q^{a}\right)}{D} \\
G_{a b} & =D^{2} \times\left(\partial_{a} \alpha^{\mu}\right)\left(\partial_{b} \alpha^{\nu}\right) g_{\mu \nu} \\
\mathcal{A}_{a} & =D \times\left(\partial_{a} \alpha^{\mu}\right) A_{\mu}
\end{aligned}
$$

where $\alpha^{\mu}$ are any convenient ( $D$ independent) functions of the coordinates $q^{a}$. Note that $G_{a b}$ differs from $g_{\mu \nu}$ transformed to $q^{a}$ coordinates by the scale factor $D^{2}$. In the same way the gauge field $\mathcal{A}_{a}$ differs from $A_{\mu}$ transformed to the coordinates $q^{a}$ by a scale factor $D$. The scale factors are chosen to scale up distances and holonomies on the patch to order unity. We also find it convenient to define the one-form field

$$
\chi_{a} \equiv D \partial_{a} \phi=\alpha_{a}^{\mu} \partial_{\mu} \phi .
$$

\footnotetext{
${ }^{25}$ See [1] for a more detailed discussion of the rational behind choosing this scaling limit.
} 
Note that $\chi_{\mu}$ is of order unity and constant (to leading order in $1 / D$ ) in scaled patch coordinates (see [1] for more discussion). The equations of motion may be rewritten in terms of scaled quantities as

$$
\begin{aligned}
\mathcal{E}_{\phi} & \equiv\left(\frac{d}{D}\right) \nabla_{a} \chi^{a}+\frac{\chi^{2}}{2}-\frac{2(d-1)}{d} e^{-\phi}-\left[\frac{D^{2}}{2 d(D-2)}\right] F_{c d} F^{c d}=0 \\
\mathcal{E}_{a b} & \equiv R_{a b}-\left(\frac{d}{D}\right)\left(\frac{\nabla_{a} \chi_{b}+\nabla_{b} \chi_{a}}{2}\right)-\left(\frac{d}{4 D^{2}}\right) \chi_{a} \chi_{b}-\frac{1}{2} F_{a c} F_{b}^{c}+g_{a b}\left[\frac{F_{c d} F^{c d}}{4(D-2)}\right]=0 \\
\mathcal{E}_{a} & \equiv \nabla_{a} F^{a b}+\frac{d}{2 D} \chi_{a} F^{a b}=0
\end{aligned}
$$

where $\nabla=$ the covariant derivative w.r.t. metric $g_{\mu \nu}$.

All quantities (curvatures, Christoffel symbols, field strengths) in (3.20) are constructed out of the scaled metric $G_{a b}$ and scaled gauge field $\mathcal{A}_{a}$.

The variables in these equations are all assumed to be of order unity. All factors of $D$ in these equations are explicit, and so the equations (3.20) are easily expanded in a power series in $1 / D$. At leading order, in particular, the equations reduce to

$$
\begin{aligned}
& \left.\mathcal{E}_{\phi}\right|_{\text {leading }} \equiv \nabla_{a} \chi^{a}+\frac{\chi^{2}}{2}-2 e^{-\phi}-\left[\frac{F_{c d} F^{c d}}{2}\right]=0, \\
& \left.\mathcal{E}_{a b}\right|_{\text {leading }} \equiv R_{a b}-\frac{\nabla_{a} \chi_{b}+\nabla_{b} \chi_{a}}{2}-\frac{1}{2} F_{a c} F_{b}^{c}=0, \\
& \left.\mathcal{E}_{a}\right|_{\text {leading }} \equiv \nabla_{a} F^{a b}+\frac{1}{2} \chi_{a} F^{a b}=0 .
\end{aligned}
$$

In this paper we search for solutions of these equations in each patch of the manifold. We require that solutions in neighbouring patches agree with each other where they overlap. We will find solutions of our equations order by order in an expansion in $\frac{1}{D}$.

\subsection{Choice of 'patch coordinates'}

In this paper we will follow [1] to implement perturbation in $1 / D$ in a patch of size $\sim \mathcal{O}\left(\frac{1}{D}\right)$ centered around an arbitrary point $x_{0}^{\mu}$ on the membrane ( $\rho=1$ surface). We will then sew together the results from each patch to obtain a global correction to the metric and gauge field in (2.5).

In order to set up the computation in any given patch, we need an explicit choice of local coordinates in each patch, i.e. an explicit choice of the coordinates $\sim\left\{y^{a}\right\}$ as defined in equation (3.18).

Having imposed $\mathrm{SO}(D-p-2)$ invariance we have three distinguished one-form fields in each patch. These one-forms are $n\left(x_{0}^{\mu}\right), u\left(x_{0}^{\mu}\right)$ and $Z\left(x_{0}^{\mu}\right)$. Note that from (3.15) it follows that

$$
Z \cdot n=0, \quad Z \cdot O=-Z \cdot u=\mathcal{O}\left(\frac{1}{D}\right),
$$

where '.' denotes contraction with respect to flat metric. 
Let $Y^{i}$ denote a set of $p$ one-form fields chosen so that

$$
Y^{i} \cdot Z=Y^{i} \cdot n=Y^{i} \cdot O=0, \quad Y^{i} \cdot Y^{j}=\delta^{i j} .
$$

There is, of course, a great deal of ambiguity in the precise details of the $Y^{i}$ fields that will play no role in what follows.

Let $\left\{x_{0}^{\mu}\right\}=\left\{w_{0}^{\alpha}, S_{0}\right\}$ represent a point on the membrane in the metric (2.5). We wish to focus on the patch of size of order $\frac{1}{D}$ around $x_{0}^{\mu}$. We set up a local coordinate system for this patch as follows.

$$
\begin{aligned}
R & =D(\rho-1), \\
V & =D\left(x^{\mu}-x_{0}^{\mu}\right) O_{\mu}\left(x_{0}\right), \\
\frac{z}{S_{0}} & =D\left(x^{\mu}-x_{0}^{\mu}\right) Z_{\mu}\left(x_{0}\right), \\
y^{i} & =D\left(x^{\mu}-x_{0}^{\mu}\right) Y_{\mu}^{i}\left(x_{0}\right) .
\end{aligned}
$$

\subsection{The perturbative metric in a patch}

In these coordinates and at leading order in the $\frac{1}{D}$ expansion, the rescaled metric and gauge field (3.18) take the form

$$
\begin{aligned}
d s^{2}= & 2\left(\frac{S_{0}}{n_{S}^{0}}\right) d R d V-\left[1-\left(1+Q_{0}^{2}\right) e^{-R}+Q_{0}^{2} e^{-2 R}\right] d V^{2} \\
& +\left[\frac{1}{1-\left(n_{S}^{0}\right)^{2}}\right] d z^{2}+\sum_{i=1}^{p} d y^{i} d y^{i}+\mathcal{O}\left(\frac{1}{d}\right), \\
e^{\phi}= & S_{0}^{2}, \\
A= & \sqrt{2} Q_{0} e^{-R} d V+\mathcal{O}\left(\frac{1}{d}\right),
\end{aligned}
$$

where $Q_{0}=Q\left(x_{0}^{\mu}\right), \quad n_{S}^{0}=\left.(n \cdot d S)\right|_{x^{\mu}=x_{0}^{\mu}}$.

Eq. (3.23) describes a configuration that is translationally invariant in the coordinates $V z$ and $y^{i}$ (but not in $R$ ). We refer to (3.23) as the black brane metric. Notice that black brane metrics are parameterized by $S_{0}, n_{S}^{0}$ and the charge $Q=Q_{0}$. Recall $r_{0}=S_{0} / n_{S}^{0}$ is the radius of the static black hole whose patch, when blown up about a membrane point with $S=S_{0}$, yields the black brane metric (3.23).

It is easily directly verified that the black brane configuration (3.23) solves the leading large $D$ equations of motion (3.21).

After appropriate scaling the metric and gauge field fluctuation at first order in $\left(\frac{1}{D}\right)$ (see (3.11)) takes the following form in the 'patch coordinates'

$$
\begin{aligned}
G_{a b}^{(1)} d q^{a} d q^{b}= & S_{(V V)} d V^{2}+2\left[\frac{S_{(V z)}}{S_{0}}\right] d V d z+\left[\frac{S_{(z z)}}{S_{0}^{2}}\right] d z^{2}+S_{(T r)} d y^{i} d y^{i} \\
& +2 V_{i}^{(V)} d y^{i} d V+2\left[\frac{V_{i}^{(z)}}{S_{0}}\right] d y^{i} d z+T_{i j} d y^{i} d y^{j} \\
\mathcal{A}_{a}^{(1)} d q^{a}= & S_{(A V)} d V+\left[\frac{S_{(A z)}}{S_{0}}\right] d z+V_{i}^{(A)} d y^{i}
\end{aligned}
$$




\subsection{The structure of perturbative equations at first order}

Let us begin the process of determining the correction to our metric and gauge field in a patch (centered about an arbitrary point on the membrane). Upon plugging first order corrected metric and gauge field into the Einstein-Maxwell equations, we find that each of these equations takes the schematic form

$$
H v^{(1)}=s^{(1)} .
$$

The term $v^{(1)}$ in (3.25) is a schematic for the collection of unknown functions in (3.11). The 'source' terms $s^{(1)}$ have their origin in the fact that a blown up patch of $(2.5)$ fails to solve the Einstein-Maxwell equations at first subleading order in $1 / D$. This failure has its roots in the following facts:

1. A patch of (2.5) differs from the black brane metric at first subleading order in $1 / D$. This difference is visible upon Taylor expanding the fields $n, u$ and $Q$ to first order about the special point $x_{0}^{\mu}$ and results in source terms proportional to the first derivative of $n u$ and $Q$.

2. The black brane itself fails to solve the Einstein- Maxwell equations at first subleading order in $1 / D$. This shows up in the fact that the equations (3.20) themselves have corrections in the $1 / D$ expansion. This gives rise to derivative free source terms.

Note that all source terms are entirely determined by the data (membrane shape, velocity field, charge field) that go into defining the ansatz metric and gauge field (2.5).

All source terms are fast varying functions of the coordinate $R$ but slow varying functions of all other coordinates. This implies that

$$
v^{(1)}=v^{(1)}\left(R, \frac{V}{D}, \frac{z}{D}, \frac{y^{i}}{D}\right),
$$

where $R$ and the other scaled coordinates are are defined in (3.22). As $v^{(1)}$ is already a fluctuation variable at order $1 / D$, derivatives of $v^{(1)}$ in all directions other than $R$ contribute to the Einstein-Maxwell equations only at order $1 / D^{2}$. It follows that the homogeneous operator $H$ is a differential operator only in the variable $R$. In other words the equations (3.25) are linear ordinary differential equations.

Even though the r.h.s. of (3.25) has its origin partly in the Taylor expansion of (2.5) about the special point $x_{o}^{\mu}$, the source functions $s^{(1)}$ in the patch about $x_{0}^{\mu}$ do not explicitly depend on the expansion coordinates $V, z, y^{i}$. The reason for this is simple. The locality of the Einstein-Maxwell equations ensures that $s^{(1)}$ is a $\frac{1}{D}$ times a local functions of the fields $\rho^{p+d}, n^{\mu}, u^{\mu}, Q$ and their derivatives. Dependence on the coordinates $V, z$ and $y^{i}$ dependence could only arise from Taylor expanding the fields $n^{\mu}, u^{\mu}$ and $Q$ about the point $x_{0}^{\mu}$. The terms proportional to $V, z, y^{i}$ in this Taylor expansion are all manifestly of order $1 / D^{2}$ or smaller. ${ }^{26}$

\footnotetext{
${ }^{26}$ On the other hand source functions have nontrivial dependence on $R$ at leading order in $1 / D$; this is a consequence of the fact that $\rho^{p+d}$ evaluates to $e^{R}$ at leading order in the large $d$ expansion, and so powers and derivatives of this function naturally appear in sources.
} 
Let us also reiterate that source $s^{(1)}$ contains at most one derivative of $n^{\mu}, u^{\mu}$ and $Q$. This follows immediately from the observation that $\rho, u$ and $Q$ are functions of $\frac{V}{D}, \frac{z}{D}$ and $\frac{y^{i}}{D}$ in the patch, and every derivative of these functions is weighted by a factor of $\frac{1}{D}$.

Let us summarize. Eq. (3.25) is a collection of an infinite number of linear ordinary differential equations in the variable $R$; one such equation at each point on the membrane world volume. At each membrane point the source functions are explicit function of $R$, with coefficients that depend on the values and (at most) one derivatives of the $\rho, u$ and $Q$ fields at that point. In to find $G_{a b}^{(1)}, \mathcal{A}_{a}^{(1)}$ and $\delta \phi^{(2)}$ we need to solve these linear differential equations at each membrane point and then sew these solutions together into a global correction to (2.5). At the technical level, the procedure for perturbation theory is strongly reminiscent of the procedure adopted in studies of the fluid gravity correspondence, see e.g. $[15,18-20]$

\subsection{Equations in the three symmetry channels}

As we have explained above, the variables in $G_{a b}^{(1)}, \mathcal{A}_{a}^{(1)}$ and $\delta \phi(1)$ consist of 7 scalar functions, 3 vector functions and one tensor function (where 'scalar', 'vector' and 'tensor' refer to the transformation property of the modes under $\mathrm{SO}(p)$ rotations in part of $x^{\mu}$ tangent space that is orthogonal to $X, n$ and $u$ ). The black brane background (3.23)), and so the operator $H$, preserves $\mathrm{SO}(p)$ symmetry. It follows that the equations (3.25) do not mix the scalar vector and tensor modes; the equations in these three sectors decouple from each other.

Tensor sector: in the tensor sector the differential equations (3.25) reduce to a single ordinary second order differential equation for a single unknown, $T_{i j}(R)$; this equation is easily solved for an arbitrary source function. We present our explicit results below.

Vector sector: in the vector sector we have four coupled equations for three unknown functions. The four equations in question are

$$
\begin{array}{rlrl}
\mathcal{E}_{R i} & =0, \quad \mathcal{E}_{V i} & =0, \\
\mathcal{E}_{z i} & =0, \quad \mathcal{E}_{i}=0,
\end{array}
$$

(see (3.20) for definitions of the equations). The directions $i$ are the $Y^{i}$ directions. They are assumed to be orthogonal to $O, u$ and $d S$.

At first order it turns out that the following linear combination of equations vanishes identically.

$$
\begin{aligned}
\partial_{R}\left[\left(\frac{S_{0}}{n_{S}^{0}}\right) \mathcal{E}_{V i}+f_{0}(R) \mathcal{E}_{R i}\right] \\
+\left[\left(\frac{S_{0}}{n_{S}^{0}}\right) \mathcal{E}_{V i}+f_{0}(R) \mathcal{E}_{R i}\right]+S_{0}\left[\frac{1-\left(n_{S}^{0}\right)^{2}}{\left(n_{S}^{0}\right)^{2}}\right] \mathcal{E}_{z i}=0,
\end{aligned}
$$

where $f_{0}(R)=1-\left(1+Q_{0}^{2}\right) e^{-R}+Q_{0}^{2} e^{-2 R}$.

We thus have only three independent vector equations for our three vector unknowns. It turns out that the the remaining three equations are easily solved for arbitrary source terms 
that obey (3.27), and in particular for the source terms that actually appear in the first order computation (see below for more details).

Scalar sector: in the scalar sector we have 11 equations for 7 variables. The 11 equations are

$$
\begin{array}{rlrlrl}
\mathcal{E}_{R R} & =0, & \mathcal{E}_{R V} & =0, & & \mathcal{E}_{R z}=0, \\
\mathcal{E}_{V V} & =0, & \mathcal{E}_{V z} & =0, & \mathcal{E}_{z z} & =0, \\
\mathcal{E}_{R} & =0, & \mathcal{E}_{V} & =0, & \mathcal{E}_{z}=0, \\
\sum_{i=1}^{p} \mathcal{E}_{i i} & =0, & \mathcal{E}_{\phi} & =0, & &
\end{array}
$$

(see (3.20) for the definition of these equations). At first order it turns out that the following four linear combination of equations automatically vanish.

Combination-1: $\partial_{R} \mathcal{E}_{R}+\mathcal{E}_{R}+\frac{\mathcal{E}_{z}}{S_{0}}=0$

Combination-2: $\partial_{R}\left[\mathcal{E}_{V V}+\left(\frac{n_{S}^{0}}{S_{0}}\right) f_{0}(R) \mathcal{E}_{R V}\right]+\left[\mathcal{E}_{V V}+\left(\frac{n_{S}^{0}}{S_{0}}\right) f_{0}(R) \mathcal{E}_{R V}\right]$

$$
-\left(n_{S}^{0}-\frac{1}{n_{S}^{0}}\right)\left[\mathcal{E}_{V z}+\frac{Q_{0} S_{0} e^{-R}}{\sqrt{2}\left[1-\left(n_{S}^{0}\right)^{2}\right]} \mathcal{E}_{R}\right]=0
$$

Combination-3: $\partial_{R}\left[\left(\frac{n_{S}^{0}}{S_{0}}\right) f_{0}(R) \mathcal{E}_{R z}+\mathcal{E}_{V z}\right]+\left[\left(\frac{n_{S}^{0}}{S_{0}}\right) f_{0}(R) \mathcal{E}_{R z}+\mathcal{E}_{V z}\right]$

$$
-\left(n_{S}^{0}-\frac{1}{n_{S}^{0}}\right) \mathcal{E}_{z z}=0
$$

Combination-4: $\partial_{R}\left[\mathcal{E}_{\phi}+2\left(\frac{n_{S}^{0}}{S_{0}}\right)^{2} f_{0}(R) \mathcal{E}_{R R}-2\left[1-\left(n_{S}^{0}\right)^{2}\right] \mathcal{E}_{z z}-\mathcal{E}_{i i}\right]$

$$
\begin{aligned}
& +2\left(\frac{n_{S}^{0}}{S_{0}}\right)^{2}\left[\partial_{R} f_{0}(R)+2 f_{0}(R)\right] \mathcal{E}_{R R}+4\left(\frac{n_{S}^{0}}{S_{0}}\right) \mathcal{E}_{R V} \\
& +4\left[\frac{1-\left(n_{S}^{0}\right)^{2}}{S_{0}}\right] \mathcal{E}_{R z}-2 \sqrt{2} Q_{0} e^{-R} \mathcal{E}_{V}=0 .
\end{aligned}
$$

We thus have exactly seven independent equations to solve for the seven unknowns in the scalar sector. It turns out that the remaining seven equations are easily solved for arbitrary sources that obey (3.29), and in particular for the source terms that actually appear in the first order computation (see below for more details).

\subsection{Basis for source functions}

Let us now turn to a description of the sources that appear on the r.h.s. of (3.25). In the scalar sector there are two kinds of sources. The first kind of source has its origin in the fact that the black brane metric (3.23) solves the Einstein-Maxwell equations only at large $D$ and not at first subleading order in $\frac{1}{D}$. This fact gives rise to sources (r.h.s. of (3.25)) that are simply functions of $R$. We also have sources from the first term in the Taylor expansion 


\begin{tabular}{|c|c|c|}
\hline $\begin{array}{c}\text { Scalars } \\
(6)\end{array}$ & $\begin{array}{c}\text { Vectors } \\
(5)\end{array}$ & $\begin{array}{c}\text { Tensors } \\
(2)\end{array}$ \\
\hline $\mathfrak{s}^{(1)}=u^{\mu} u^{\nu} K_{\mu \nu}$ & $\mathfrak{v}_{\mu}^{(1)}=u^{\nu} P_{\mu}^{\alpha} K_{\nu \alpha}$ & $\mathfrak{t}_{\mu \nu}^{(1)}=P_{\mu}^{\alpha} P_{\nu}^{\beta}\left[\frac{K_{\alpha \beta}}{2}-\left(\frac{\mathfrak{s}_{3}}{p}\right) \eta_{\alpha \beta}\right]$ \\
$\mathfrak{s}^{(2)}=u^{\mu} Z^{\nu} K_{\mu \nu}$ & $\mathfrak{v}_{\mu}^{(2)}=u^{\nu} P_{\mu}^{\alpha} \partial_{\nu} u_{\alpha}$ & $\mathfrak{t}_{\mu \nu}^{(2)}=P_{\mu}^{\alpha} P_{\nu}^{\beta}\left[\frac{\partial_{\alpha} u_{\beta}+\partial_{\beta} u_{\alpha}}{2}-\left(\frac{\mathfrak{s}_{4}}{p}\right) \eta_{\alpha \beta}\right]$ \\
$\mathfrak{s}^{(3)}=P^{\mu \nu} K_{\mu \nu}$ & $\mathfrak{v}_{\mu}^{(3)}=P_{\mu}^{\alpha}(Z \cdot \partial) u_{\alpha}$ & \\
$\mathfrak{s}^{(4)}=P^{\mu \nu} \partial_{\mu} u_{\nu}$ & $\mathfrak{v}_{\mu}^{(4)}=P_{\mu}^{\alpha} \partial_{\alpha} Q$ & \\
$\mathfrak{s}^{(5)}=u^{\mu} \partial_{\mu} Q$ & $\mathfrak{v}_{\mu}^{(5)}=Z^{\nu} P_{\mu}^{\alpha} K_{\nu \alpha}$ & \\
$\mathfrak{s}^{(6)}=Z^{\mu} Z^{\nu} K_{\mu \nu}$ & & \\
\hline
\end{tabular}

Table 1. Data at 1 st order in $\frac{1}{D}$ expansion.

of the functions $n_{\mu}, u^{\mu}$ and $Q$ expanded about $x_{0}^{\mu}$. Let $\mathfrak{s}^{(a)}\left(a=1 \ldots N_{S}\right)$ denote the set of scalar first derivatives of the functions $n, u$ and $Q$. Let $\mathfrak{s}_{0}=1$ (this allows us to deal with the first kind of source mentioned above). On general grounds, the source $\mathcal{S}_{m}$ terms in the $m$ th scalar equations $E_{m}^{S}$ takes the form

$$
\mathcal{S}_{m}=\sum_{a=0}^{N_{S}} \mathcal{S}_{m}^{a}(R) \mathfrak{s}^{(a)} .
$$

In a similar manner we let $\mathfrak{v}^{(a)}\left(a=1 \ldots N_{V}\right)$ denote the set of scalar first derivatives of the functions $n, u$ and $Q$. The source terms $\mathcal{V}_{m i}$ in the $m$ th vector equation $E_{m i}^{V}$ take the form

$$
\mathcal{V}_{m i}=\sum_{a=1}^{N_{V}} \mathcal{V}_{m}^{a}(R) \mathfrak{v}_{i}^{(a)} .
$$

Finally if $\mathfrak{t}_{i j}^{(a)}\left(a=1 \ldots N_{T}\right)$ denote the set of tensor first derivatives of the functions $n, u$ and $Q$, then the source terms $\mathcal{T}_{i j}$ in the unique tensor equation must take the form

$$
\mathcal{T}_{i j}=\sum_{a=1}^{N_{T}} \mathcal{T}^{a}(R) \mathfrak{t}_{i j}^{(a)} .
$$

It turns out at first order $\left(N_{S}=6, \quad N_{V}=5, \quad N_{T}=2\right)$. In table (1) we have listed and explicit basis for independent scalar vector and tensor data at first order. Here $P_{\mu \nu}$ is the projector perpendicular to $u_{\mu}, n_{\mu}$ and $Z_{\mu}$.

\subsection{Equations of motion from regularity at the horizon}

We are interested in solutions to the equations of perturbation theory that are everywhere regular (away from the black hole singularity that will turn out to be shielded by an event horizon). Even though all our source functions are regular, this condition is not automatic at $R=0$ (i.e. $\rho=1$ ). This perhaps surprising fact plays a key role in this paper. This subsection is devoted to a more detailed exposition of this fact. 
Let $E^{M N}$ denote the Einstein equation obtained by varying the Einstein-Maxwell Lagrangian w.r.t. $g_{M N}$, and let $M_{N}$ denote the Maxwell equation obtained by varying the Einstein-Maxwell Lagrangian w.r.t. $A_{M}$. As we have explained above, the perturbative procedure of this paper is geared to determining the $\rho$ dependence of unknown metric and gauge field components. For our purposes it is thus natural to view the $\rho$ direction as a Euclidean 'time' direction in which we wish to understand 'dynamics'. From this point of view the equations

$$
C_{\mathrm{Ein}}^{M}=E^{M N}(d \rho)_{M}=E^{M \rho}, \quad C_{\mathrm{Max}}=M^{N}(d \rho)_{N}=M^{\rho},
$$

are, respectively, the Einstein and Maxwell 'constraint' equations.

From the 'geometrical' viewpoint (see below for much more discussion) $\left[C_{\text {Ein }}\right]_{M}$ could be decomposed into two scalars and one vector equation while $C_{\mathrm{Max}}$ is a scalar equation. However perturbative procedure described so far is not geometrical: it treats the isometry directions as special. From our current point of view $\left[C_{\text {Ein }}\right]_{M}$ may be decomposed into three $\mathrm{SO}(p)$ invariant scalars $\left[C_{\text {Ein }} \cdot d \rho\right],\left[C_{\text {Ein }} \cdot V\right]$ and $\left[C_{\text {Ein }} \cdot z\right]$ and an $\mathrm{SO}(p)$ vector $\left(C_{\text {Ein }}^{M}\right.$ projected orthogonal to $n^{\mu}, u^{\mu}$ and $\left.Z^{\mu}\right)$.

In the scalar sector it is easily verified that

$$
\begin{aligned}
\left(C_{\text {Einp }} \cdot Z\right) & \propto\left[\left(\frac{S_{0}}{n_{S}^{0}}\right) \mathcal{E}_{V z}+f_{0}(R) \mathcal{E}_{R z}\right] \\
& \propto f_{0}(R)^{2} \frac{d}{d R}\left[\frac{S_{(V z)}(R)}{f_{0}(R)}\right]+\Sigma_{(V z)}(R)=0 \\
C_{\mathrm{Max}} & \propto \mathcal{E}_{R} \\
& \propto f_{0}(R)\left(\frac{d}{d R} S_{(A z)}(R)\right)+\sqrt{2} Q_{0} e^{-R} S_{(V z)}(R)+\Sigma_{(A z)}(R)=0 .
\end{aligned}
$$

Here $\Sigma_{(V z)}(R)$ the full source term for the combination of equations $\left[\left(\frac{S_{0}}{n_{S}^{0}}\right) \mathcal{E}_{V z}+f_{0}(R) \mathcal{E}_{R z}\right]$ while $\Sigma_{(A z)}(R)$ is the source term in $\mathcal{E}_{R} \cdot{ }^{27}$

An inspection of (3.34) reveals that this equation admits nonsingular solutions at $R=0$ if and only if the linear term, in the Taylor expansion of $\Sigma_{(V z)}(R)$ about $R=0$, vanishes. Provided this condition is met the solution to (3.34) is nonsingular. Once this condition is met it follows from (3.34) that

$$
S_{(V z)}(R=0)=\frac{\Sigma_{(V z)}(R=0)}{f^{\prime}(R=0)} .
$$

Turning to the equation (3.35), it is easily seen that the solution to this equation is nonsingular if and only if $\left[\sqrt{2} Q_{0} e^{-R} S_{(V z)}(R)+\Sigma_{(A z)}(R)\right]$ vanishes at $R=0$. Using (3.36), this condition is equivalent to the requirement that $\left[\sqrt{2} Q_{0} \frac{\Sigma_{(V z)}(R=0)}{f^{\prime}(R=0)}+\Sigma_{(A z)}(R=0)\right]$ vanish. Plugging in the explicit expressions for the source functions $\Sigma_{(A z)}(R)$ and $\Sigma_{(A z)}(R)$

\footnotetext{
${ }^{27}$ Clearly, each of $\Sigma_{(V Z)}(R)$ and $\Sigma_{(A z)}(R)$ are linear combinations of the previously defined quantities $\mathcal{S}_{i}^{a}(R)$.
} 
we find that we have nonsingular solutions if and only if

$$
\begin{aligned}
& (X-u) \cdot K \cdot(X-u)-\left[\frac{2 Q^{2}}{\left(1-Q^{2}\right)}\right][(X-u) \cdot K \cdot u]=\left(\frac{1-n_{S}^{2}}{S n_{S}}\right), \\
& (X-u) \cdot \partial Q=Q[(X-u) \cdot K \cdot u],
\end{aligned}
$$

where $X=\frac{d S}{n_{S}}-n=\left(\frac{n_{S}}{S}\right) Z$.

In the vector sector, the projection of $C_{\text {Einp }}$ may be shown to be proportional to

$$
\left[\left(\frac{S^{0}}{n_{S}^{0}}\right) \mathcal{E}_{V i}+f_{0}(R) \mathcal{E}_{R i}\right] \propto f_{0}(R) \frac{d}{d R}\left[V_{i}^{(z)}(R)\right]+\mathcal{V}_{i}^{(Z)}(R)=0 .
$$

Here $\mathcal{V}_{i}^{(Z)}(R)$ is the combination of source terms in the first line of (3.38) - and so an appropriate linear combination of $\mathcal{V}_{m i}^{a}(R)$ This equation has regular solutions if $\mathcal{V}_{i}^{(Z)}(R)$ vanishes at $R=0$ i.e. if

$$
P_{j}^{i}\left[(X-u) \cdot \partial(u-n)_{i}+Q^{2}\left(X \cdot \partial n_{i}-u \cdot \partial u_{i}\right)\right]=0,
$$

$$
\text { where } X=\frac{d S}{n_{S}}-n=\left(\frac{n_{S}}{S}\right) Z \text {. }
$$

It may be verified that (3.37) and (3.39) exhaust the constraints of regularity; once these equations hold the solution for the first order correction to the black brane metric and gauge field can always be chosen (by choosing appropriate integration constants in the solutions of the differential equation) to be regular at $R=0$ (and everywhere else within the patch).

In summary, the perturbative procedure described in this subsection yields regular solutions if and only if the equations of motion (3.37) and (3.39) are obeyed.

\subsection{Equivalence to the equations of [1] in the uncharged limit}

Note that the same null one-form $O_{\mu}$ has been parametrized in a different way in [1].

$$
O=A\left(d S-u^{\text {there }}\right)=n-u^{\text {here }},
$$

where $u^{\text {there }}$ is the velocity field used in [1] and in this subsection, $u^{\text {here }}$ will denote the velocity field we used in this paper. Recall that $u^{\text {there }}$ was chosen to obey $u^{\text {there }} \cdot d S=0$. Dotting (3.40) with $d S$ we find $A=n_{S}=n \cdot d S$ from which it follows that

$$
u^{\text {there }}=\frac{u^{\text {here }}-n}{n_{S}}+d S .
$$

This is the reason the equations of motion for the uncharged membrane as reported in equation (1.7) of [1] apparently do not match with the $Q \rightarrow 0$ limit of the equations of motion we derived in (3.37) and (3.39). However, we shall see that once we take into account this difference in the definition of $u$, the uncharged limit of our equations of motion exactly matches with that of $[1]$. 
The equations of motion for the uncharged membrane were reported in equation (1.7) of [1] as

$$
\begin{aligned}
& U_{\perp} \cdot K \cdot U_{\perp}+n_{S}\left(n_{S}^{2}-1\right) / S=0, \\
& \left(\left(U_{\perp} \cdot \nabla\right) u^{\text {there }}\right) \cdot P_{\text {there }}^{\mu \nu}=0, \\
& U_{\perp}=U-(U \cdot n) n, \quad U=d S+n_{S}^{2}\left(d S-u_{\mu}^{\text {there }} d x^{\mu}\right) .
\end{aligned}
$$

The projector $P_{\text {there }}^{\mu \nu}$ projects orthogonal to the subspace spanned by $u^{\text {there }}, n$ and $d S$. But $u_{\text {there }}$ is a linear combination of $u_{\text {here }}$ and $n$. Therefore it follows that the projector $P_{\text {there }}^{\mu \nu}$ employed in (3.42) agree with the projector $P^{\mu \nu}$ in (3.39). The covariant derivative ' $\nabla$ ' is a derivative defined in the auxiliary space. In our choice of coordinates, this could be replaces by ' $\partial$ '.

Using (3.41) we could express the vector $U_{\perp}$ in (3.42) in terms of the velocity $u^{\text {here }}$

$$
\begin{aligned}
& U_{\perp}=U-2 n_{S} n=d S-n_{S}\left(u^{\text {here }}+n\right)=n_{S}\left(X-u^{\text {here }}\right), \\
& \text { where } X=\frac{d S}{n_{S}}-n=\left(\frac{n_{S}}{S}\right) Z .
\end{aligned}
$$

Substituting equation (3.41) and (3.43) in (3.42), we find ${ }^{28}$

$$
\begin{array}{r}
\left(X-u^{\text {here }}\right) \cdot K \cdot\left(X-u^{\text {here }}\right)+\frac{n_{S}^{2}-1}{S n_{S}}=0, \\
{\left[\left(\left(X-u^{\text {here }}\right) \cdot \partial\right)\left(n-u^{\text {here }}\right)\right] \cdot P=0 .}
\end{array}
$$

Equations (3.44) exactly match with the $(Q \rightarrow 0)$ limit of equations (3.37) and (3.39).

\subsection{Conditions to fix the integration constants}

As we have explained above, the first order corrections to (2.5) are obtained by solving a collection of linear ordinary differential equations at each point on the membrane. As mentioned above these equations turn out to be explicitly solvable and yield regular solutions provided the equations of motion of subsection 3.10 are obeyed. The solutions to these equations are, however, not yet unique. as they depend on as yet undetermined integration constants at each membrane point. As we have mentioned in the previous subsection, some of these constants are determined by the requirement of regularity at $R=0$. This condition however leaves several integration constants undetermined. ${ }^{29}$ In order to obtain a unique solution to our equations we will impose additional physically motivated constraints that will uniquely determine these integration constants.

\footnotetext{
${ }^{28}$ note that the projected derivative of $\frac{u_{\text {here }}-n}{n_{S}}$ equals $\frac{1}{n_{S}}$ times the projected derivative of $u-n$ as the term with $n_{S}$ differentiated vanishes under projection. Where $u$ is the new velocity.

${ }^{29}$ As the integration 'constants' can, in general, be unconstrained functions of the membrane world volume (they are constants only in that they do not depend on $R$ ) they are in fact undetermined integration functions on the membrane world volume.
} 
Asymptotic flatness: an obvious requirement that we impose is that the correction metric and gauge field $g_{\mu \nu}^{(1)}$ and $A_{\mu}^{(1)}$ vanish exponentially rapidly as $R \rightarrow \infty$. This condition ensures that the full spacetime metric rapidly approaches the metric of flat space upon moving a large distance (in units of $\frac{1}{D}$ ) away from the membrane. This condition sets the value of several integration constants.

Normalization conditions: even after imposing the condition of asymptotic flatness, it turns out that we still have two undetermined integration constants in the scalar sector and one in the vector sector. This is precisely as should be expected on physical grounds. Our starting spacetime (2.5) was parameterized by two scalar functions (the shape of the membrane and its charge density field) plus one vector function (the velocity field). A redefinition of these fields (e.g. $Q \rightarrow Q+\mathcal{O}(1 / D)$ leaves (2.5) unchanged at leading order, but modifies it at first subleading order. Such a redefinition will modifies the first order correction to the metric by a compensating amount. For this reason we should expect the first order correction to have a two parameter ambiguity in the scalar sector and a one parameter ambiguity in the vector sector, precisely as we find. ${ }^{30}$

The ambiguity described above is a result of the fact that we have not yet supplied a precise all orders definition of the shape, velocity and charge density fields that enter into the leading order solution (2.5). Such a definition may be supplied by specifying an additional constraint on all higher order corrections to (2.5) that would fix the field redefinition ambiguity described in the previous paragraph. In this paper we choose to do this by requiring that $S_{(V V)}, V_{\mu}^{(V)}$ and $S_{(A V)}$ all vanish at $R=0$. More invariantly we impose the condition that

$$
H_{M N} n^{N}=A_{M} n^{M}=0 \text { when } \rho=1 .
$$

We refer to these additional conditions - that effectively define the shape, velocity and charge density fields - as 'normalization' conditions.

It may be checked (see the upcoming paper [14]) that the normalization conditions we have chosen ensure, in particular that the surface $\rho=1$ is a null surface which we will later identify with the event horizon of the spacetime.

The conditions of asymptotic flatness together with the normalization conditions are sufficient to fix all integration constants, and yield unique expressions for the first order correction the the metric and gauge field (2.5).

\subsection{Results for the first order correction on the patch}

In this subsection we present the explicit solution for the metric and the gauge field corrections at first order in $\mathcal{O}\left(\frac{1}{D}\right)$. Our explicit results are presented for $(p=2)$, but will be generalized to all $p$ in the next section. As mentioned above, our solution takes the form (3.24). In the rest of this subsection we present our explicit results for the functions that appear in $(3.24)^{31}$

\footnotetext{
${ }^{30} \mathrm{~A}$ very similar issue arose in the study of the fluid gravity correspondence, and was dealt with in a manner similar to that described below. See e.g. [15, 18-20].

${ }^{31}$ The solution presented in this subsection depends on three functions $Q, S$ and $n_{S}$. Strictly speaking they should be written as $Q_{0}, S_{0}$ and $n_{S}^{0}$, the values of these functions at $x^{\mu}=x_{0}^{\mu}$. But we did not write it
} 


\subsubsection{The functions appearing in the gauge field}

$$
\begin{aligned}
V_{i}^{A}(R)= & -\sqrt{2} Q\left(\frac{S}{n_{S}}\right)^{2}\left[\left(1-Q^{2}\right) \mathfrak{v}_{i}^{(5)}+\left(1+Q^{2}\right)\left(\frac{n_{S}}{S}\right) \mathfrak{v}_{i}^{(2)}\right] R e^{-R} \\
& +\sqrt{2} Q^{3}\left(\frac{S}{n_{S}}\right)^{2}\left(\mathfrak{v}_{i}^{(5)}-\left(\frac{n_{S}}{S}\right) \mathfrak{v}_{i}^{(2)}\right)\left[1+\log \left(1-Q^{2} e^{-R}\right)\right] e^{-R} \\
S_{(A z)}(R)= & -\left[\frac{2 \sqrt{2} S^{2} Q^{3} e^{-R}}{\left(1-n_{S}^{2}\right)\left(1-Q^{2}\right)}\right]\left[1+\log \left(1-Q^{2} e^{-R}\right)\right] \mathfrak{s}_{1} \\
& +\left[\frac{2 \sqrt{2} S^{3} Q e^{-R}}{n_{S}\left(1-n_{S}^{2}\right)\left(1-Q^{2}\right)}\right]\left[\left(Q^{2}-R+Q^{2} R\right)+Q^{2} \log \left(1-Q^{2} e^{-R}\right)\right] \mathfrak{s}_{2} \\
S_{(A V)}(R)= & \sqrt{2} Q R e^{-R}\left(\frac{S}{n_{S}}\right)\left(\frac{\mathfrak{s}^{(5)}}{Q}-\mathfrak{s}^{(1)}+\frac{S}{n_{S}} \mathfrak{s}^{(2)}\right) \\
& +2 \sqrt{2}\left(\frac{Q^{3}}{1-Q^{2}}\right) e^{-R} \Upsilon_{A}(R)\left(\frac{S}{n_{S}}\right)\left(\mathfrak{s}^{(1)}-\frac{S}{n_{S}} \mathfrak{s}^{(2)}\right)
\end{aligned}
$$

where

$$
\Upsilon_{A}(R)=\int_{0}^{R} d x \log \left(1-Q^{2} e^{-x}\right) .
$$

\subsubsection{The functions appearing in the metric}

$$
\begin{aligned}
T_{i j}(R)= & \left(\frac{2 S}{n_{S}}\right)\left(\mathfrak{t}_{i j}^{(1)}-\mathfrak{t}_{i j}^{(2)}\right) \log \left(1-Q^{2} e^{-R}\right) . \\
V_{i}^{(z)}(R)= & {\left[\frac{S^{2}\left(1+Q^{2}\right)}{n_{S}\left(1-n_{S}^{2}\right)}\right]\left(\mathfrak{v}_{i}^{(5)}-\left(\frac{n_{S}}{S}\right) \mathfrak{v}_{i}^{(2)}\right) \log \left(1-Q^{2} e^{-R}\right) } \\
V_{i}^{(V)}(R)= & \left(\frac{Q S}{n_{S}}\right)^{2}\left[1-e^{-R}-f_{0}(R)\left(1+\log \left[1-Q^{2} e^{-R}\right]\right)\right]\left(\mathfrak{v}_{i}^{(5)}-\left(\frac{n_{S}}{S}\right) \mathfrak{v}_{i}^{(2)}\right) \\
& -R\left[1-f_{0}(R)\right]\left(\frac{S}{n_{S}}\right)^{2}\left[\left(1-Q^{2}\right)_{i}^{(5)}+\left(1+Q^{2}\right)\left(\frac{n_{S}}{S}\right) \mathfrak{v}_{i}^{(2)}\right] \\
S_{(V z)}(R)= & S_{(V z)}^{(1)}(R) \mathfrak{s}^{(1)}+S_{(V z)}^{(2)}(R) \mathfrak{s}^{(2)}, \\
S_{(V z)}^{(1)}(R)= & -\left[\frac{2 Q^{2} S^{2}}{\left(1-n_{S}^{2}\right)\left(1-Q^{2}\right)}\right]\left[Q^{2}\left(e^{-R}-e^{-2 R}\right)-f_{0}(R) \log \left(1-Q^{2} e^{-R}\right)\right], \\
S_{(V z)}^{(2)}(R)= & {\left[\frac{2 Q^{2} S^{3}}{n_{S}\left(1-n_{S}^{2}\right)\left(1-Q^{2}\right)}\right]\left[\left(e^{-R}-e^{-2 R}\right)\left(Q^{2}-R+Q^{2} R\right)\right.} \\
& \left.-f_{0}(R) \log \left(1-Q^{2} e^{-R}\right)\right]-\frac{2 S^{3} R e^{-R}}{n_{S}\left(1-n_{S}^{2}\right)} . \\
S_{(z z)}(R)= & {\left[\mathfrak{s}^{(2)}-\left(\frac{n_{S}}{S}\right) \mathfrak{s}^{(1)}\right]\left[\frac{2 S^{4}\left(1+Q^{2}\right)}{\left(1-n_{S}^{2}\right)^{2}\left(1-Q^{2}\right)}\right] \log \left(1-Q^{2} e^{-R}\right) . }
\end{aligned}
$$

that way firstly because of notational simplicity and secondly because we know that the difference is always suppressed by terms of order $\mathcal{O}\left(\frac{1}{D}\right)$. 


$$
\begin{aligned}
S_{(T r)}(R)= & {\left[-2+\left(\frac{S}{n_{S}}\right)\left(\mathfrak{s}^{(3)}-\mathfrak{s}^{(4)}\right)\right] \log \left(1-Q^{2} e^{-R}\right) . } \\
S_{(V V)}(R)= & -\sqrt{2} Q e^{-R} S_{A V}(R)+Q^{2}\left[e^{-2 R}-e^{-R}\right] \\
& +2 e^{-R}\left[Q^{2} R\left(\frac{\mathfrak{s}^{(5)}}{Q}-\mathfrak{s}^{(1)}+\frac{S}{n_{S}} \mathfrak{s}^{(2)}\right)+\Upsilon_{H}(R)\left(\mathfrak{s}^{(1)}-\frac{S}{n_{S}} \mathfrak{s}^{(2)}\right)\right],
\end{aligned}
$$

where

$$
\begin{aligned}
f_{0}(R) & =1-\left[\left(1+Q^{2}\right) e^{-R}-Q^{2} e^{-2 R}\right], \\
\Upsilon_{H}(R) & =\left[\left(e^{-R}-Q^{2}\right) \log \left(1-Q^{2} e^{-R}\right)-\left(1-Q^{2}\right) \log \left(1-Q^{2}\right)+\left(\frac{Q^{2}\left(1+Q^{2}\right)}{1-Q^{2}}\right) \Upsilon_{A}(R)\right]
\end{aligned}
$$

\subsubsection{Correction (2nd order in $\frac{1}{D}$ ) to the scalar field $\phi$}

$$
\begin{aligned}
\delta \phi & =\sum_{k=1}\left(\frac{1}{D}\right)^{k} \delta \phi^{(k)} \\
\delta \phi^{(1)} & =0 \\
\delta \phi^{(2)}(R) & =-2 S_{(T r)}(R)-\left(\frac{1-n_{S}^{2}}{S^{2}}\right) S_{(z z)}(R) .
\end{aligned}
$$

\subsubsection{The $Q \rightarrow 0$ limit}

If we set $Q$ to zero in equation (3.45) to (3.57), most of the functions vanish except $V_{i}^{(V)}$ and $S_{(V z)}$. In the uncharged limit, the metric takes the following simple form,

$$
\left.G_{a b}^{(1)} d q^{a} d q^{b}\right|_{\text {uncharged }}=-2 R e^{-R}\left[\left(\frac{S}{n_{S}}\right)^{2}\left(\mathfrak{v}_{i}^{(5)}+\frac{n_{S}}{S} \mathfrak{v}_{i}^{(1)}\right) d y^{i}+\frac{S^{3} \mathfrak{s}^{(2)}}{n_{S}\left(1-n_{S}^{2}\right)} d z\right] d V
$$

\subsection{The global first order metric}

With the first order corrected patch metric in hand (see the previous subsection), it is straightforward to find the global form of the metric and gauge field which, when expanded in any patch around a membrane point, will reproduce the results of appendix 3.13. In order to obtain this global form we simply make the replacements

$$
e^{R} \rightarrow \rho^{-D}, \quad R \rightarrow D \times(\rho-1), \quad d V \rightarrow O_{M} d x^{M}, \quad d R \rightarrow D \times(d \rho),
$$

in the results of subsection 3.13. The final metric obtained in this manner is already reasonably compact. There is, however, a physically motivated rewriting of this result in a form that is both more elegant and also makes manifest the 'geometrical' nature of our final result, as we explain in more detail in the next section. 


\section{Geometrical form of the first order corrected metric}

\subsection{Redistribution invariance and the geometrical form}

The membrane equations (3.37) and (3.39) make make special reference to $e^{\phi}, n_{S}$ and the one-form field $Z_{\mu}$. The same is true of our explicit results for the first order correction to (2.5), presented in subsection 3.13. Expressions involving $S, n_{S}$ and $Z$ of course are only well defined for configurations that preserve an $\mathrm{SO}(D-p-2)$ symmetry. Moreover the definition of, e.g. $S$ depends on the details of the isometry.

Unconstrained dependence on $S$ and $n_{S}$ is unacceptable for the following reason. A solution that preserves an $\mathrm{SO}(D-p-2)$ isometry also preserves an $\mathrm{SO}\left(D-p^{\prime}-2\right)$ isometry for all $p^{\prime}>p$. It follows that any solution of the equations for a particular choice of $p$ must also be a solution of the same equation for all larger $p$. We refer to this requirement as the requirement of redistribution invariance.

The requirement of redistribution invariance is most simply met if the equation of motion and the metric and gauge field can both be written in an explicitly geometrical form that makes no reference to the particular isometry group of the solution. The membrane equation and first order metric and gauge field obtained in this section do indeed turn out to have this property.

The reader may, at first, wonder how it is possible for expressions with explicit appearances of $S$ and $n_{S}$ to also be geometrical. This is, infarct, possible in the large $D$ limit, as we now explain with an example. Consider the manifestly geometrical expression $\nabla^{2} \rho$ where $\nabla$ refers to the covariant derivative on the full flat $D$ dimensional embedding spacetime. Let us now evaluate this expression in the large $D$ limit restricting attention to membrane configurations that preserve an $\mathrm{SO}(D-p-2)$ isometry. The computation is most conveniently performed using the following coordinates

$$
d s^{2}=\eta_{\alpha \beta} d w^{\alpha} d w^{\beta}+d S^{2}+S^{2} d \Omega_{d}^{2},
$$

in the embedding flat space. Using these coordinates

$$
\nabla^{2} \rho=\frac{1}{S^{d}} \partial_{\mu}\left(S^{d} \partial^{\mu} \rho\right)
$$

At leading order in the large $D$ limit this expression reduces to $D \frac{d S \cdot d \rho}{S}$ It follows that $\frac{d S \cdot d \rho}{S}=\frac{\nabla^{2} \rho}{D}$ at leading order in the large $D$ limit. Consequently any appearance of $\frac{d S \cdot d \rho}{S}$ in any equation may be explicitly geometrized.

Similar manipulations allow us to geometrize several other expressions involving $S$, $n_{S}$ and $Z$. Of course not every expression involving these quantities can be geometrized (expressions that are not redistribution invariant certainly cannot). However it turns out that all terms in the equations of motion (3.37) and (3.39) and all terms in our explicit expression for the metric and gauge field in subsection 3.13 can be geometrized. The final geometrical expressions for equations of motion and the the first order corrected metric and gauge field are more compact than the unprocessed expressions. In the next section we present our final results for the first order corrections to the metric and gauge field in explicitly geometrical form. In the subsequent subsection we do the same for the equations of motion. 


\subsection{Metric and gauge field in geometric form}

While we expect the first order correction to the metric and gauge field to be geometrizable on physical grounds, this requirement is nontrivial at the algebraic level. The vector $Z_{\mu}-$ which is treated as a special in the computation described above and in subsection 3.13 has no intrinsic geometrical significance. ${ }^{32}$ If the first order correction to the metric and gauge field is completely geometrical, it should be possible to rewrite it in a manner that makes no reference to $Z_{\mu}$. In fact it should be possible to rewrite the metric and gauge field in the form

$$
\begin{aligned}
h_{M N} & =F(\rho) O_{M} O_{N}+H_{M N}^{(T)}+2 O_{(M} H_{N)}^{(V)}+H^{(S)} O_{M} O_{N}+H^{(T r)} \mathcal{P}_{M N}, \\
A_{M} & =\sqrt{2} Q \rho^{-(D-3)} O_{M}+\left(A^{(S)} O_{M}+A_{M}^{(V)}\right)
\end{aligned}
$$

where

$$
\begin{aligned}
F(\rho) & =\left[\left(1+Q^{2}\right) \rho^{-(D-3)}-Q^{2} \rho^{-2(D-3)}\right], \\
\mathcal{P}_{M N} & =\eta_{M N}-O_{M} n_{N}-O_{N} n_{M}+O_{M} O_{N}, \\
\mathcal{P}^{M N} H_{N}^{(V)} & =H^{(V) M}, \quad \mathcal{P}^{M N} A_{N}^{(V)}=A^{(V) M}, \quad \mathcal{P}^{M N} H_{M Q}^{(T)}=H_{Q}^{(T) N}, \quad \mathcal{P}^{M N} H_{M N}^{(T)}=0,
\end{aligned}
$$

Eq. (4.1) should reproduce the expressions for $g_{\mu \nu}, A_{\mu}$ (see (3.11)) as well as the scalar $\phi$ (recall that $\phi$ is part of the full $D$ dimensional metric).

The general metric and gauge field presented in (4.1) are parameterized by three unknown scalar functions (rather than the seven scalar functions in (3.11) and in the expansion of the scalar $\phi$ ) and by two vector functions (rather than three vector functions in (3.11)). It follows that the explicit results of subsection 3.13 can be recast into the form (4.1) only if the seven scalar functions determined in subsection 3.13 obey four constraints, and the three vector functions determined in the same appendix obey a single constraint equation.

We have verified that our explicit results do infarct obey all constraints. We view this fact as an impressive consistency check of the complicated algebra that went into obtaining the explicit results of subsection 3.13.

As our explicit results obey all consistency conditions, it is possible to rewrite our final results in the explicitly geometric form (4.1). We find that the various free functions in (2.7) are given by

$$
\begin{aligned}
A_{M}^{(V)}= & -\left(\frac{\sqrt{2}}{D}\right) Q \rho^{-D}\left[D(\rho-1)\left(\mathfrak{V}_{(1)}-Q^{2} \mathfrak{V}_{(2)}\right)-Q^{2}\left[1+\log \left(1-\rho^{-D} Q^{2}\right)\right] \mathfrak{V}_{(2)}\right]_{M} \\
& +\mathcal{O}\left(\frac{1}{D}\right)^{2} \\
A^{(S)}= & \left(\frac{1}{D}\right)\left[\sqrt{2} Q D(\rho-1) \rho^{-D} \mathfrak{S}_{(1)}+2 \sqrt{2}\left(\frac{Q^{3}}{1-Q^{2}}\right) \rho^{-D} \Upsilon_{A}(\rho) \mathfrak{S}_{(2)}\right] \\
& +\mathcal{O}\left(\frac{1}{D}\right)^{2} .
\end{aligned}
$$

\footnotetext{
${ }^{32}$ On the other hand the vectors $n^{\mu}$ and $u^{\mu}$ are intrinsically geometrical, as they describe the membrane shape and velocity field in $D$ dimensional spacetime.
} 


\begin{tabular}{|l|c|}
\hline Scalars & $\mathfrak{S}_{(1)}=\left(\frac{D}{\mathcal{K}}\right)\left[\frac{u \cdot \partial Q}{Q}-u \cdot K \cdot u+\frac{(u \cdot \partial) \mathcal{K}}{\mathcal{K}}\right]$ \\
& $\mathfrak{S}_{(2)}=\left(\frac{D}{\mathcal{K}}\right)\left[u \cdot K \cdot u-\frac{(u \cdot \partial) \mathcal{K}}{\mathcal{K}}\right]$ \\
\hline Vectors & $\mathfrak{V}_{(1)}^{M}=\left(\frac{D}{\mathcal{K}}\right)\left[\frac{\nabla_{N} \mathcal{K}}{\mathcal{K}}+(u \cdot \nabla) u_{N}\right] \mathcal{P}^{N M}$ \\
& $\mathfrak{V}_{(2)}^{M}=\left(\frac{D}{\mathcal{K}}\right)\left[\frac{\nabla_{N} \mathcal{K}}{\mathcal{K}}-(u \cdot \nabla) u_{N}\right] \mathcal{P}^{N M}$ \\
\hline Tensor & $\mathfrak{T}^{M N}=\mathcal{P}^{M Q_{1}}\left(\frac{D}{\mathcal{K}}\right)\left[\frac{\nabla_{Q_{1}} O_{Q_{2}}+\nabla_{Q_{2}} O_{Q_{1}}}{2}-\eta_{Q_{1} Q_{2}}\left(\frac{\nabla \cdot O}{D-2}\right)\right] \mathcal{P}^{Q_{2} N}$ \\
\hline
\end{tabular}

Table 2. We list the data that enters into our explicit results for the first order correction to the metric and the gauge field. All data is presented in explicitly geometrical form. $\rho, Q$ and $u^{\mu}$ should be thought of as two functions and a vector field in flat $D$ dimensional space. All derivatives that appear in this table are covariant derivatives w.r.t. flat $D$ dimensional space.

$$
\begin{aligned}
H_{M N}^{(T)}= & \left(\frac{2}{D}\right) \log \left(1-Q^{2} \rho^{-D}\right) \mathfrak{T}_{M N}+\mathcal{O}\left(\frac{1}{D}\right)^{2}, \\
H_{M}^{(V)}= & \left(\frac{1}{D}\right)\left\{Q^{2}\left[\left(F(\rho)-\rho^{-(D-3)}\right)+(F(\rho)-1) \log \left(1-Q^{2} \rho^{-D}\right)\right] \mathfrak{V}_{(2) M}\right. \\
& \left.\quad-D(\rho-1) F(\rho)\left[\mathfrak{V}_{(1)}-Q^{2} \mathfrak{V}_{(2)}\right]_{M}\right\}+\mathcal{O}\left(\frac{1}{D}\right)^{2} . \\
H^{(S)}= & -\sqrt{2} Q \rho^{-D} A^{(S)}+\left(\frac{1}{D}\right)\left[\rho^{-(D-3)}-F(\rho)\right] \\
& \quad+\left(\frac{2}{D}\right) \rho^{-D}\left[Q^{2} D(\rho-1) \mathfrak{S}_{(1)}+\Upsilon_{H}(\rho) \mathfrak{S}_{(2)}\right]+\mathcal{O}\left(\frac{1}{D}\right)^{2}, \\
H^{(T r)}= & \mathcal{O}\left(\frac{1}{D}\right)^{3},
\end{aligned}
$$

where

$$
\begin{aligned}
F(\rho) & =\left[\left(1+Q^{2}\right) \rho^{-(D-3)}-Q^{2} \rho^{-2(D-3)}\right], \\
\Upsilon_{A}(\rho) & =\int_{0}^{D(\rho-1)} d x \log \left(1-Q^{2} e^{-x}\right), \\
\Upsilon_{H}(\rho) & =\left[\left(\rho^{D}-Q^{2}\right) \log \left(1-Q^{2} \rho^{-D}\right)-\left(1-Q^{2}\right) \log \left(1-Q^{2}\right)+Q^{2}\left(\frac{1+Q^{2}}{1-Q^{2}}\right) \Upsilon_{A}(\rho)\right] .
\end{aligned}
$$

\subsubsection{The limit $Q \rightarrow 0$}

The results of the previous subsection simplifies drastically in the limit $Q \rightarrow 0$. In this limit the gauge field simply vanishes, and the full first order corrected metric is given by the remarkably simple expression

$$
\begin{aligned}
d s_{\text {uncharged }}^{2}= & d s_{\text {flat }}^{2}+\rho^{-(D-3)}\left(O_{M} d x^{M}\right)^{2} \\
& -2(\rho-1) \rho^{-(D-3)}\left[\mathfrak{V}_{(1)}\right]_{M} O_{N} d x^{M} d x^{N}+\mathcal{O}\left(\frac{1}{D}\right)^{2},
\end{aligned}
$$

where $\mathfrak{V}_{1}$ is defined in table 2 . 
In appendix $\mathrm{B}$ we have shown how this geometric form of the metric and gauge field reduce to the solution presented in subsection 3.13, once we impose the constraint of $\mathrm{SO}(D-p-2)$ invariance on all geometric data.

\subsection{Geometrizable form of the membrane equations of motion}

The membrane equations of motion (3.37) and (3.39) may be recast into a simpler looking form. We have a combined equation capturing both vector equation and one of the scalar equations.

$$
\begin{array}{r}
{\left[(u-X) \cdot \tilde{\nabla} O-Q^{2}(u \cdot \tilde{\nabla}) u+Q^{2}(X \cdot K)\right] \cdot \mathcal{P}+\left(\frac{n_{S}}{S}\right)\left(1-Q^{2}\right) X=0} \\
(X-u) \cdot \tilde{\nabla} Q+Q\left[\left(\frac{S}{n_{S}}\right)(u \cdot \tilde{\nabla})\left(\frac{n_{S}}{S}\right)-(u \cdot K \cdot u)\right]=0
\end{array}
$$

where

$$
\begin{gathered}
\mathcal{P}_{\mu \nu}=\text { Projector perpendicular to } u_{\mu} \text { and } n_{\mu}, \\
X=\frac{d S}{n_{S}}-n=\left(\frac{S}{n_{S}}\right) Z, \quad O=n-u
\end{gathered}
$$

In this equation $\tilde{\nabla}$ above is the partial derivative on the membrane world volume viewed as a submanifold of (3.12).

The projection of equation (4.7) perpendicular to $Z_{\mu}$ directly reduces to the vector equation of motion as given in equation (3.39). In appendix $C$ we have shown that equation (4.8) is equal to second equation of (3.37). Moreover the dot product (4.7) with $Z_{\mu}$ equals the first equation in (3.37) upto correction of $\mathcal{O}\left(\frac{1}{D}\right)$.

We re emphasize that the projector employed in (4.7) projects orthogonal to $n$ and $u$ but not to $Z_{\mu}$. In other words (4.7) unifies a $\mathrm{SO}(p)$ scalar and $\mathrm{SO}(p)$ vector equation into a single 'geometrical' vector equation. This fact may lead the reader to suspect that the equations (4.7) and (4.8) are geometrizable (i.e. can be written without any explicit reference to the isometry direction. This is indeed the case. It is not too difficult to demonstrate that the geometric form of the equations of motion, (1.1) (see the introduction) reduce immediately to (4.7) and (4.8) upon using the dictionary of translation as presented in appendix D.

\subsubsection{Constraint equations and the membrane equations of motion}

In the previous section we explained that the Einstein and Maxwell constraint equations play a special role in our construction. We obtained the membrane equations of motion from the requirement that these bulk equations admit nonsingular solutions. Once the membrane equations were imposed, it was possible to utilize the constraint equations to solve for some unknown bulk scalar and vector fields in terms of others in a nonsingular manner. We have already mentioned in subsection 3.10 that the geometric nature of the membrane equations is a direct consequence of the geometric nature of Einstein's constraint equations.

In this subsection we wish to focus on the fact the constraint equations played two roles in the perturbative program of the previous section. 
1. They yielded the membrane equations of motion.

2. They allowed us to solve for some unknowns bulk fields in terms of others.

Interestingly enough, the relations that we obtain from item (2) above are all automatic in the expression (4.1). In other words the relations of item (2) above are simply a subset of the relations between various unknown bulk vectors fields and various unknown bulk scalar scalars fields that are forced on us once we assume that the first order metric and gauge field correction take on a geometric form.

Had we used hindsight to set up our perturbative expansion in a manifestly geometric manner by simply assuming that our first order correction takes the form (4.1) then the constraint equations of subsection 3.10 would simply have reduced to the membrane equations (1.1), exactly as in the studies of the fluid gravity correspondence (see e.g. [15-20]).

Recall that the Einstein constraint equations assert the conservation of the Brown York Stress tensor, while the Maxwell constraint equation asserts the conservation of a 'charge current' $F^{\rho M}$. These observations suggest that it may be possible to recast our membrane equations (1.1) as conservation equations for a manifestly geometric membrane stress tensor and charge current, as was the case in the study of fluid gravity. We will not pursue this point further in this paper but hope to return to it in the near future.

\subsection{Comparison with the Reissner-Nordstrom solution}

As an elementary check of the results reported in subsection 4.2, consider the following membrane configuration. Let $u_{M} d x^{M}=-d t, Q=$ const and let the membrane surface be given by $x^{M}\left(\eta_{M N}+u_{M} u_{N}\right) x^{N}=r_{0}^{2}$. It is easily verified that this configuration solves the membrane equations (1.1); clearly this static soap bubble solution is dual to the ReissnerNordstrom black hole (2.2).

We will now use the formalism developed in this paper to determine the spacetime metric and gauge field dual to this membrane solution, to first order in $1 / D$.

Let us first start with the leading order solution (2.5) dual to this solution. We need to find a function $\rho$ that obeys the first of equation (2.6) and s.t. $\rho=1$ on the membrane surface listed above. The unique solution to this mathematical problem is given by

$$
\rho=\sqrt{\frac{x^{M}\left(\eta_{M N}+u_{M} u_{N}\right) x^{N}}{r_{0}^{2}}} .
$$

Next we must determine the spacetime fields $u$ and $Q$ fields that reduce to $-d t$ and $q$ on the membrane and obey (2.6) everywhere in the bulk of $D$ dimensional flat space. The unique solution to this problem is given by $u=-d t$ and $Q$ in all of flat space. The leading order solution with this data is given by (2.5) with these choices for $\rho, Q$ and $u$.

Let us now turn to the first order correction. It is easily verified that relevant geometric data as given in table 2 vanish on this particular profile of $\rho$ and $u_{M}$ and $Q$. It follows that the first order correction to the gauge field vanishes. The first order correction to the metric also almost vanishes. Of all the quantities listed in (4.2), (4.3) and (4.4) vanish except for $H^{S}$ which evaluates to $\left(\frac{1}{D}\right)\left[\rho^{-(D-3)}-F(\rho)\right]$ with $F(\rho)$ listed in (4.5). 
Plugging these values of $\rho, u_{M}$ and $Q$ into (2.5) and adding the correction terms (4.1) , it follows that the metric and gauge field dual to our simple solution of the membrane equations is given, to first order in $\frac{1}{D}$, by

$$
\begin{aligned}
g_{M N}= & \eta_{M N}+\left[\left(1+Q^{2}\right) \rho^{-(D-3)}-Q^{2} \rho^{-2(D-3)}\right] O_{M} O_{N} \\
& +\frac{Q^{2}}{D}\left(\rho^{-2(D-3)}-\rho^{-(D-3)}\right) O_{M} O_{N}+\mathcal{O}\left(\frac{1}{D}\right)^{2},
\end{aligned}
$$

eq. (4.9) is easily seen to agree with the exact RN black hole solution (2.2) expanded to leading nontrivial order in $1 / D$. The function $\rho$ of (2.2) agrees exactly with the function $\rho$ reported above. The only appearance of $D$ in the solution (2.2) is in the factor $c_{D}$. Upon plugging the expansion

$$
c_{D}=1-\frac{1}{D}+\mathcal{O}\left(\frac{1}{D}\right)^{2},
$$

into (2.2) we immediately recover (4.9).

The matching performed in this subsection was almost trivial. In the upcoming paper [14] we use the same method to match the metric dual to a rigidly rotating solution of the membrane equations to the much more complicated exact solution of an uncharged rotating Myers Perry black hole [21]. Once again we find a perfect match between the two expansions.

\section{Light quasinormal spectrum of the RN black hole}

Our membrane equations (1.1) should describe all $\mathrm{SO}(D-p-2)$ invariant black hole dynamics (over times scales much larger than $1 / D$ ) at large $D$. As a first application of these equations, in this section we will use them to obtain a prediction for the spectrum of light quasinormal modes (those with frequencies of order unity rather than of order $D$ ) about charged Reissner-Nordstrom black holes in the large $D$ limit.

For the purposes of this section we work in polar coordinates in $D$ spacetime dimensions. Our coordinate system for flat space is given by

$$
d s^{2}=-d t^{2}+d r^{2}+r^{2} d \Omega_{D-2}^{2} .
$$

The exact solution of (1.1) dual to RN black holes was presented in subsection 4.4. In the coordinates (5.1) this solution takes the particularly simple form

$$
r=1, \quad Q=Q_{0}=\text { const }, \quad u=-d t,
$$

where we have chosen units that set the size of the membrane to unity. ${ }^{33}$

\footnotetext{
${ }^{33}$ We do not loose generality by making this choice. The classical Einstein Maxwell equations studied in this paper enjoy invariance under the following 'scaling' symmetry:

$$
\tilde{g}_{M N}=\alpha^{2} g_{M N}, \quad \tilde{F}_{M N}=\alpha F_{M N} .
$$

This scale transformation together with the coordinate change $\tilde{x}^{M}=\alpha x^{M}$ transforms a Reissner Nordstrom black hole with Schwarzschild radius $r_{0}$ and charge parameter $Q_{0}$ into a Reissner Nordstrom black hole with Schwarzschild radius $\alpha r_{0}$ and charge parameter $Q_{0}$. It follows that the quasinormal mode frequencies of the black hole parameterized by $\left(r_{0}, Q_{0}\right)$ are simply $\frac{1}{r_{0}}$ times those for the black hole parameterized by $\left(1, Q_{0}\right)$. For this reason we will perform all computations in this section with black holes of radius unity, and simply reinsert factors of $r_{0}$ in the final answer.
} 
The most general linearized perturbation around (5.2) takes the form

$$
\begin{aligned}
r & =1+\epsilon \delta r(t, \theta), \\
Q & =Q_{0}+\epsilon \delta Q(t, \theta), \\
u & =-d t+\epsilon \delta u_{\mu}(t, \theta) d x^{\mu} .
\end{aligned}
$$

We now adopt the following strategy. We simply insert the expansions (5.3) into (1.1), work to linear order in $\epsilon$ and obtain the effective linear equations for the fluctuation fields $\delta r, \delta Q$ and $\delta u_{\mu}$ defined in (5.3). These fields live on the membrane world volume. A useful set of coordinates on this world volume are the angular coordinates $\theta^{a}$ on $\Omega_{D-2}$ and time. The metric on the membrane world volume in these coordinates is obtained by inserting the first of (5.3) into (5.1), and is given in terms of the function $\delta r(t, \theta)$. To linear order in $\epsilon$ the metric on the membrane surface is given by

$$
d s^{2}=-d t^{2}+(1+2 \epsilon \delta r) d \Omega_{D-2}^{2} .
$$

It is useful to have a dictionary to go between forms and vectors that live on the membrane and those that live in spacetime. Consider a vector field $A^{\mu}$ that lives on the membrane. This vector field may be uplifted to spacetime. The spacetime components $A_{(\mathrm{ST})}^{M}$ of this vector field are given in terms of the world volume components $A^{a}$ by the formulae

$$
A_{(\mathrm{ST})}^{a}=A^{a}, \quad A_{(\mathrm{ST})}^{t}=A^{t}, \quad A_{(\mathrm{ST})}^{r}=\epsilon\left(A^{t} \partial_{t} \delta r+A^{a} \partial_{a} \delta r\right)
$$

In a similar manner, a one-form field in spacetime $B_{a}^{(\mathrm{ST})}$ is easily pulled back to a one-form field $B_{a}$ on the membrane. In formulae

$$
B_{a}=B_{a}^{(\mathrm{ST})}+\epsilon B_{r}^{(\mathrm{ST})} \partial_{a} \delta r, \quad B_{t}=B_{t}^{(\mathrm{ST})}+\epsilon B_{r}^{(\mathrm{ST})} \partial_{t} \delta r .
$$

As a simple consistency check on these formulae, it is easily verified that $A^{\mu} B_{\mu}=$ $A_{(\mathrm{ST})}^{M} B_{M}^{(\mathrm{ST})}$. Below we will treat the field $u_{\mu}$ in (5.3) as a one-form field on the membrane. Recall that $u_{\mu}$ is constrained by the requirement $\nabla \cdot u=0$, i.e. that the velocity field is divergence free. Here $\nabla$ is the covariant derivative taken in the metric (5.4).

In order to evaluate the terms in (1.1) we need to compute the, normal one-form and extrinsic curvature of the membrane as well as a few derivatives of the velocity field. The computations involved are straightforward: to linear order in $\epsilon$ we find

$$
\begin{aligned}
n_{r} & =1 \\
n_{\mu} & =-\epsilon \partial_{\mu} \delta r \\
K_{t t} & =-\epsilon \partial_{t}^{2} \delta r \\
K_{t a} & =-\epsilon \partial_{t} \nabla_{a} \delta r \\
K_{a b} & =-\epsilon \nabla_{a} \nabla_{b} \delta r+(1+\epsilon \delta r) g_{a b}, \\
\delta u_{t} & =0, \quad(u \cdot u=-1)
\end{aligned}
$$




$$
\begin{aligned}
(u \cdot K)_{t} & =K_{t t}=-\epsilon \partial_{t}^{2} \delta r \\
(u \cdot K)_{a} & =-\epsilon \partial_{t} \nabla_{a} \delta r+\epsilon \delta u_{a}, \\
\mathcal{K} & =K_{A}{ }^{A}=D\left(1-\epsilon\left(1+\frac{\nabla^{2}}{D}\right) \delta r\right),
\end{aligned}
$$

where $a, b$ are angular directions on $\Omega_{D-2}$, the symbol $\mu$ runs over these angular coordinates and time (i.e $\mu=(t, a)$ ) and $g_{a b}$ is the round metric on $S^{D-2}$. All indices in (5.7) are indices on the spherical metric world volume, i.e. on a space with metric

$$
d s^{2}=-d t^{2}+d \Omega_{D-2}^{2},
$$

and all covariant derivatives in (5.7) are taken in the background spacetime (5.9).

Using (5.7), the first equation in (1.1) may be shown to reduce, at linearized order in $\epsilon$, to

$$
\left(1+\frac{\nabla^{2}}{D}\right) \delta u_{a}+\left(1-Q_{0}^{2}\right) \nabla_{a}\left(1+\frac{\nabla^{2}}{D}\right) \delta r-\partial_{t} \nabla_{a} \delta r-\left(1+Q_{0}^{2}\right) \partial_{t} \delta u_{a}=0 .
$$

(All covariant derivatives are once again evaluated on the metric (5.9)).

As we have noted above, the fluctuation velocity field $\delta u_{a}$ is constrained by the condition $\nabla \cdot u=0$. The divergence in this equation is evaluated in the full membrane metric. Rewriting this constraint in terms of fields that are taken to propagate on the fixed metric (5.9) (to linear order in $\epsilon$ and leading order in $D$ ) we find

$$
\nabla_{\mu} \delta u^{\mu}=-D \partial_{t} \delta r
$$

with the covariant derivatives now evaluated on the metric (5.9). From now on until the end of this section our fluctuation fields will all be taken to propagate on the fixed background (5.9) and all covariant derivatives will refer to this metric unless explicitly otherwise declared.

As $\delta u^{t}$ vanishes (this follows upon linearizing the equation $u \cdot u=-1$ ), (5.11) may be rewritten as

$$
\nabla_{a} \delta u^{a}=-D \partial_{t} \delta r
$$

In order to solve this equation it is useful to define

$$
\delta u_{a}=\nabla_{a} \Phi+\delta v_{a}
$$

where

$$
\nabla \cdot \delta v=0
$$

It follows from (5.12) that

$$
\nabla^{2} \Phi=-D \partial_{t} \delta r .
$$

Below we will use this equation to eliminate $\Phi$ in favour of $\delta r$. Note that (5.15) admits a solution if and only if its r.h.s. has no overlap with the kernel of the operator $\nabla^{2}$. As the kernel of $\nabla^{2}$ consists of functions that are constant on the sphere, it follows that (5.15) is 
consistent if and only if the spatially constant (i.e. $l=0$ mode) of $\delta r$ is time independent. When this condition is obeyed, $\Phi$ may be solved for in terms of $\delta r$, as we will do below.

Plugging the expansion (5.13) into the equation (5.10) we find

$$
\begin{aligned}
\left(1+\frac{\nabla^{2}}{D}-\left(1+Q_{0}^{2}\right) \partial_{t}\right) \delta v_{a}= & -\left(\left(1-Q_{0}^{2}\right) \nabla_{a}\left(1+\frac{\nabla^{2}}{D}\right)-\partial_{t} \nabla_{a}\right) \delta r \\
& -\left(1+\frac{\nabla^{2}}{D}-\left(1+Q_{0}^{2}\right) \partial_{t}\right) \nabla_{a} \Phi .
\end{aligned}
$$

\subsection{The spectrum of shape fluctuations}

Taking the divergence of (5.16) and using (5.14) and (5.15) we obtain the following decoupled scalar equation for the fluctuation ${ }^{34}$ field $\delta r$

$$
D\left(1+Q_{0}^{2}\right) \partial_{t}^{2} \delta r-2 D\left(1+\frac{\nabla^{2}}{D}\right) \partial_{t} \delta r+\left(1-Q_{0}^{2}\right)\left(1+\frac{\nabla^{2}}{D}\right) \nabla^{2} \delta r=0
$$

The most general linearized fluctuation $\delta r$ can be expanded as

$$
\delta r=\sum_{l, m} a_{l m} Y_{l m} e^{-i \omega_{l}^{r} t} .
$$

Here $Y_{l m}$ are spherical harmonics on $S^{D-2}, l$ labels the spherical harmonic representation, $m$ is a collective label for all the internal quantum numbers within a given spherical harmonic representation.

Let us pause to give a more complete description of scalar spherical harmonics in arbitrary dimensions, and in particular to compute the eigenvalue under $\nabla^{2}$ acting on the $l^{\text {th }}$ spherical harmonic. The $l^{\text {th }}$ spherical harmonic, $Y_{l m}$, are composed of the collection of functions on $S^{D-2}$ obtained by restricting homogeneous degree $l$ polynomials in $R^{D-1}$ to the unit sphere. The polynomials in questions are linear combinations of monomials of the form $a_{\mu_{1} \mu_{2} \mu_{3} \ldots \mu_{l}} x^{\mu_{1}} x^{\mu_{2}} \ldots x^{\mu_{l}}$ where $a_{\mu_{1} \mu_{2} \mu_{3} \ldots \mu_{l}}$ are symmetric and traceless tensors. It is easily shown that ${ }^{35}$

$$
-\nabla_{S^{D-2}}^{2} Y_{l m}=l(D+l-3) Y_{l m} .
$$

\footnotetext{
${ }^{34}$ In order to obtain (5.18) we have used and

$$
\begin{aligned}
\nabla^{a} \nabla^{2} \delta u_{a} & =\nabla^{2} \nabla_{a} \delta u^{a}+R^{a b} \nabla_{a} \delta u_{b} \\
& =\nabla^{2} \nabla_{a} \delta u^{a}+D g^{a b} \nabla_{a} \delta u_{b}, \\
& =D\left(1+\frac{\nabla^{2}}{D}\right) \nabla_{a} \delta u^{a}=-D^{2}\left(1+\frac{\nabla^{2}}{D}\right) \partial_{t} \delta r .
\end{aligned}
$$
}

${ }^{35}$ This may be demonstrated as follows. The condition of tracelessness ensures that the degree $l$ polynomials described above obey the equation $\nabla^{2} \Phi=0$, where $\nabla^{2}$ is evaluated in $R^{D-1}$. But

$$
0=\nabla_{R^{D-1}}^{2} \Phi=\frac{1}{r^{D-2}} \partial_{r}\left(r^{D-2} \partial_{r} r^{l}\right)+\frac{\nabla_{S^{D-2}}^{2} \Phi}{r^{2}} .
$$

(the r.h.s. of this equation is $\nabla^{2}$ of the function in $R^{D-1}$ evaluated in polar coordinates). Here $\nabla_{S^{D-2}}^{2}$ is the Laplacian evaluated on the unit sphere. Eq. (5.21) follows from (5.20). 
Plugging the expansion (5.19) into (5.18) and using (5.21) we find (at leading order in large $D$ )

$$
\omega_{l}^{r}=\frac{-i(l-1) \pm \sqrt{(l-1)\left(1-l Q_{0}^{4}\right)}}{1+Q_{0}^{2}} .
$$

Re inserting factors of $r_{0}$ (see the discussion in the introduction to this section) we find (5.21) we find (at leading order in large $D)^{36}$

$$
r_{0} \omega_{l}^{r}=\frac{-i(l-1) \pm \sqrt{(l-1)\left(1-l Q_{0}^{4}\right)}}{1+Q_{0}^{2}} .
$$

(5.23), our final result for the light quasinormal mode frequencies associated with shape fluctuations, is correct as stated for $l>1$, but requires clarification for in special cases $l=0$ and $l=1$ for the reasons we now describe.

Let us first consider $l=0$. In this case (5.23) predicts the existence of quasinormal modes with frequencies $\omega r_{0}=0$ and $\omega r_{0}=\frac{2 i}{1+Q_{0}^{2}}$. As noted under (5.15), however, modes at $l=0$ are physical only if they are time independent. It follows that we have only one mode at $l=0$ : this mode has $\omega=0 .{ }^{37}$ This zero mode has a simple physical interpretation; it corresponds to an infinitesimal uniform rescaling of the black hole radius.

Let us now turn to $l=1$. In this case we have a degeneracy of quasinormal mode frequencies; both modes have $\omega=0$. The formula (5.23) was obtained by assuming harmonic dependence in time and solving for the harmonic frequencies, and it is well known that this procedure requires modification in the case that the frequencies are degenerate. In order to see how this works, we note that the specialization of (5.18) to modes with $l=1$ yields the very simple equation

$$
\partial_{t}^{2} \delta r=0
$$

It follows that the two solutions to this equation are $\delta r=Y_{1}^{m}\left(a_{m}+b_{m} t\right)$ where $a_{m}$ and $b_{m}$ are arbitrary constants. These two zero modes also have a simple physical interpretation. The mode multiplying $a_{m}$ is an infinitesimal translation of the black hole, while $b_{m}$ parameterizes an infinitesimal boost of the black hole. Note that the $m$ labels for $l=1$ scalar spherical harmonics are precisely the labels for a vector in $(D-1)$ dimensions, as appropriate for translations and boosts.

As we have mentioned above, (5.23) is correct as stated for $l \geq 2$. It is easily verified $^{38}$ that all quasinormal modes with $l \geq 2$ have negative imaginary components (and so represent decaying fluctuations).

\footnotetext{
${ }^{36} \mathrm{~K}$. Tanabe has informed us that he is also studying the dynamics of charged black holes at large $D$ and has independently obtained the result (5.23).

${ }^{37} \mathrm{Had}$ the mode with $\omega r_{0}=\frac{2 i}{1+Q_{0}^{2}}$ been physical, it would have represented an instability, contradicting the well known stability of Reissner Nordstrom black holes (atleast of sufficiently small charge) in arbitrary dimensions.

${ }^{38}$ Note that a Reissner Nordstrom black hole with $Q_{0}=1$ is extremal at large $D$. All regular black holes have $\left|Q_{0}\right|<1$.
} 


\subsection{The spectrum of velocity fluctuations}

The fact that the shape fluctuation $\delta r$ obeys the equation of the previous subsection ensures that the r.h.s. of (5.16) vanishes. The velocity fluctuations, $\delta v_{a}$, are thus effectively constrained to obey (5.16) with its r.h.s. set to zero.

The fluctuation field $\delta v$ may be expanded in vector spherical harmonics

$$
\delta v_{a}=\sum_{l, m} b_{l m} Y_{a}^{l m} e^{-i \omega_{l}^{v} t}
$$

Let us pause to describe vector spherical harmonics in arbitrary dimension in more detail. The $l^{t h}$ vector spherical harmonic may be obtained as a restriction of a vector field on $R^{D-1}$ to the unit sphere. The vector field in question is made up as a linear sum of vector valued monomials of the form $V_{\mu \mu_{1} \mu_{2} \ldots \mu_{l}} x^{\mu_{1}} x^{\mu_{2}} \ldots x^{\mu_{l}}$ where $V_{\mu \mu_{1} \mu_{2} \ldots \mu_{l}}$ is traceless, symmetric in all of its indices except the first one, and it is zero when it's first index is symmetrized with any of the others. In particular, tracing the first index of $b$ with any of the others gives zero.

It follows that each of the vector valued monomials listed above obeys the equations

$$
\nabla \cdot V=0, \quad \nabla^{2} V=0
$$

where the covariant derivatives are taken in the flat space $R^{D-1}$. The restriction of each of these vector valued monomials to the unit sphere yields a vector field tangent to the unit sphere (this is because the $r$ component of these vector fields - proportional to the monomial with first index dotted with $x^{\mu}$ - vanishes). Let this vector field be denoted by $V$. It is easily verified that $\nabla \cdot V=0$ (where the covariant derivative is now taken on the unit sphere). We demonstrate in appendix $\mathrm{E}$ that

$$
\nabla^{2} V=-[(D+l-3) l-1] V
$$

where, in this equation, $V$ is viewed as a vector field on the unit sphere and $\nabla$ is the covariant derivative on the unit sphere.

Plugging the expansion (5.25) into (5.16) and setting the coefficient of every independent vector spherical harmonic to zero we obtain, at leading order in large $D$

$$
\omega_{l}^{v}=\frac{-i(l-1)}{1+Q_{0}^{2}} .
$$

This formula agrees with the formula for the spectrum of vector quasinormal modes presented in [1] in the limit $Q_{0} \rightarrow 0$. Reinstating factors of $r_{0}$ we have

$$
r_{0} \omega_{l}^{v}=\frac{-i(l-1)}{1+Q_{0}^{2}} \quad(l=1,2,3 \ldots)
$$

Note that all the velocity quasinormal modes are pure (negative) imaginary, and so represent a ring down that decays without oscillations. Vector harmonics with $l=1$ are zero modes. These modes transform in the representation $(1,1,0,0, \ldots 0)$ - i.e. the adjoint representation - of $\mathrm{SO}(D-1)$ and have a simple physical interpretation. These zero modes turn on an infinitesimal spin on the for the black holes, i.e. begin to take one along the branch of the large $D$ version of Kerr Newman black holes. 


\subsection{The spectrum of charge fluctuations}

The spectrum of charge fluctuations is governed by the second of (1.1), which we repeat here for convenience

$$
\frac{\nabla^{2} Q}{\mathcal{K}}-u \cdot \nabla Q=Q\left(\frac{u \cdot \nabla \mathcal{K}}{\mathcal{K}}-u \cdot K \cdot u\right)
$$

Plugging (5.3) into this equation we obtain the linearized equation

$$
\left(\frac{\nabla^{2}}{D}-\partial_{t}\right) \delta Q=Q_{0}\left(\partial_{t}^{2}-\partial_{t}\left(\frac{\nabla^{2}}{D}+1\right)\right) \delta r
$$

Plugging in the expansion

$$
\delta Q=\sum_{l, m} q_{l m} Y_{l m}(\theta) e^{-i t \omega_{l}^{Q}}
$$

and focusing on the coefficient of $Y_{l m}$ for a particular value of $l$, the r.h.s. of (5.31) is a source term which drives $\delta Q$ at the frequency $\omega_{l}^{r}$ given by (5.42). A source of the form

$$
\delta r=\sum_{l, m} a_{l m} Y_{l m}(\theta) e^{-i \omega_{l}^{r} t}
$$

induces the response

$$
\delta Q_{f}=\sum_{l, m} a_{l m} \frac{i \omega_{l}^{r} Q_{0}\left(l-1-i \omega_{l}^{r}\right)}{l-i \omega_{l}^{r}} Y_{l m}(\theta) e^{-i \omega_{l}^{r} t}
$$

The most general solution of (5.31) is given by a linear sum of the particular solution (5.34) and the most general solution to the homogeneous equation, i.e. to the equation (5.31) with the r.h.s. set to zero. In order to determine the quasinormal frequencies we associated with $Q$ oscillations we solve for the frequencies of these homogeneous modes. This is easily accomplished. Using (5.21) we find, at leading order in large $D$,

$$
-l+i \omega_{l}^{Q}=0
$$

which gives the QN frequency for the charge perturbations

$$
\omega_{l}^{Q}=-i l
$$

Reinstating factors of $r_{0}$ we have

$$
r_{0} \omega_{l}^{Q}=-i l
$$

As in the case of velocity fluctuations, the charge fluctuation quasinormal modes are pure negative imaginary, and so represent diffusive decay without oscillation. $\omega$ vanishes when $l=0$. The corresponding zero mode is simply an infinitesimal uniform rescaling of $Q_{0}$. 


\subsection{A consistency check for shape fluctuations}

The spectrum of shape fluctuations can be rederived starting from the equation (1.2), i.e.

$$
\left(1-Q^{2}\right)\left[\frac{\nabla^{2} \mathcal{K}}{\mathcal{K}^{2}}-\frac{u \cdot \nabla \mathcal{K}}{\mathcal{K}}\right]=\left(1+Q^{2}\right)\left(\frac{u \cdot \nabla \mathcal{K}}{\mathcal{K}}-u \cdot K \cdot u\right) .
$$

The linearized equation is given by

$$
\left(1-Q_{0}^{2}\right)\left(\partial_{t}-\frac{\nabla^{2}}{D}\right)\left(1+\frac{\nabla^{2}}{D}\right) \delta r=\left(1+Q_{0}^{2}\right)\left(\partial_{t}^{2}-\partial_{t}\left(1+\frac{\nabla^{2}}{D}\right)\right) \delta r .
$$

Now let's consider the perturbation in membrane shape function to be a particular mode, namely

$$
f(t, \theta)=\sum_{l, m} a_{l m} Y_{l m}(\theta) e^{-i \omega_{l}^{r} t} .
$$

This turns (5.39) into an algebraic equation for a given mode

$$
\left(\omega_{l}^{r}\right)^{2}+\frac{2 i \omega_{l}^{r}(l-1)}{1+Q_{0}^{2}}-\frac{1-Q_{0}^{2}}{1+Q_{0}^{2}} l(l-1)=0,
$$

which has roots

$$
\omega_{l}^{r}=\frac{-i(l-1) \pm \sqrt{(l-1)\left(1-l Q_{0}^{4}\right)}}{1+Q_{0}^{2}} .
$$

They exactly match with (5.22).

Recall that we have argued above that the divergence of (1.1) agrees with (1.2) for all configurations that preserve an $\mathrm{SO}(D-p-2)$ isometry. In this subsection we have shown, however, the spectrum of shape fluctuations computed from the divergence of (1.1) agrees with the spectrum computed from (1.2) even though arbitrary spherical harmonics do not, in general, preserve a large isometry subgroup. The reason this had to work is as follows. In any spherical harmonic representation there exist special spherical harmonics that preserve a large isometry group. It follows from our general arguments above the two equations considered in this subsection must give the same spectrum of shape fluctuations for these special modes. However the equations analyzed in this subsection are geometrical, and in particular respect the full $\mathrm{SO}(D-1)$ rotational symmetry group of the background solution, and so generate the same spectrum of oscillations for all spherical harmonics in a given representation.

In summary the two equations had to give the same spectrum for some particular elements of the spherical harmonic representation. Rotational invariance then forces them to give the same spectrum for all spherical harmonics in the same representation, as we actually find.

\section{Discussion}

In this paper we have presented a construction of a large class of solutions of the EinsteinMaxwell equations. Our solutions are in one to one correspondence with the solutions of 
the equations of a charged, nongravitational membrane propagating in flat space according to the dynamical equations (1.1).

We have used our membrane equations to generate a prediction for the spectrum of light quasinormal modes about Reissner-Nordstrom black holes in Einstein-Maxwell gravity. As a check on our results it would be useful to independently compute these quasinormal mode frequencies, perhaps using the gauge invariant formulation of [22].

All of the computations presented in this paper have been performed at first nontrivial order in the expansion in $1 / D$. It is of great interest to generalize the computations presented herein to the next order in this expansion. Apart from determining second order corrections to the membrane equations presented in this paper such a computation would allow us to distinguish between different geometrical presentations of the first order equations (e.g the equation (1.2) and the divergence of (1.1)) (see the introduction for a discussion).

In this paper we have derived equations of membrane dynamics assuming that our configuration preserves an $\mathrm{SO}(D-p-2)$ isometry. As we have explained above, however, our final results are geometrical (in that they make no reference to the isometry algebra and treat all dimensions democratically). It is possible that the final geometrical equations are valid in more general situations, i.e. for configurations that preserve no isometry but perhaps obey some other weaker conditions. ${ }^{39}$ It would be interesting to investigate this further.

In order to gain intuition for the membrane equations derived in this paper, it would be useful to determine and study the properties of a class of simple solutions of these equations. In the upcoming paper [14] we will present a detailed study of stationary solutions to the membrane equations (1.1). As we have mentioned in the introduction, this allows us to make contact between the membrane equations presented in this paper and the membrane analysis of static and stationary black holes at large $D$ presented in $[9,10]$.

It would also be interesting to follow the lead of [11-13] and attempt to investigate Gregory-Laflamme type instabilities using an appropriate extension of the framework presented in this paper.

The solutions presented in this paper rapidly approach empty flat space away from their event horizons. At every order in the expansion in $1 / D$ the gauge field and metric simply vanishes far away from the membrane. Non perturbatively in $1 / D$ (most likely at order $1 / D^{D}$ ) we expect our membrane motions to excite a Maxwell and gravitational radiation field. As this radiation field is the means by which an external observer can actually observe the black hole dynamics described in this paper, it is of great interest to find the formula that determines this field. We hope to return to this question in the near future.

On a related note, any extended object that consistently sources gravity and Maxwell radiation should possess a conserved charge current and stress tensor. It would be interesting to find all orders formulae (within the $1 / D$ expansion) for the charge current and stress tensor of the membrane.

\footnotetext{
${ }^{39}$ We thank A. Strominger for a question about this possibility.
} 
Once all these issues have been settled satisfactorily, it would of course be interesting to simulate complicated dynamical processes (e.g. black hole collisions) using our membrane equations, and compare our results with numerical simulations in $D=4$. Such a comparison would throw light on the question of whether the beautiful structures that emerge in black hole dynamics at large $D$ are also a useful starting point for a perturbative expansion for the dynamics of astrophysical black holes.

\section{Acknowledgments}

We would like to thank A. De and A. Saha for collaboration in the initial stages of this work and S. Das, Y. Dandekar, R. Emparan, K. Inbasekar, S. Mazumdar, A. Singh, A. Strominger and S. Trivedi for useful discussions. We would also like to thank T. Takayanagi and K. Tanabe for comments on a preliminary version of this manuscript. S.B. would like to acknowledge the hospitality of the University of Barcelona while this work was in progress. S.M. would like to acknowledge the hospitality of the Institute of Mathematical Sciences, Chennai in the final stages of this work. The work of S.B. was supported by an India Israel (ISF/UGC) joint research grant. The work of M.M, S.M and S.T was supported by a separate India Israel (ISF/UGC) grant, as well as the Infosys Endowment for the study of the Quantum Structure of Space Time. We would all also like to acknowledge our debt to the people of India for their steady and generous support to research in the basic sciences.

\section{A Reissner-Nordstrom solution in Kerr-Schild coordinates}

The static Reissner-Nordstrom black hole solution is very familiar. This solution is most usually presented in Schwarzschild like coordinates. In these coordinates the spacetime manifestly Minkowskian at infinity. However the coordinates are singular at the black hole horizon. Let $\tilde{t}$ be the Schwarzschild time coordinate. The coordinate change

$$
d \tilde{t}=d v-\frac{d r}{f(r)}
$$

recasts the solution as

$$
\begin{aligned}
& d s^{2}=2 d v d r-f(r) d v^{2}+r^{2} d \Omega_{D-2}^{2}, \\
& A=\sqrt{2} Q\left(\frac{r_{0}}{r}\right)^{D-3} d v \\
& f(r)=1-\left(1+Q^{2} c_{D}\right)\left(\frac{r_{0}}{r}\right)^{D-3}+c_{D} Q^{2}\left(\frac{r_{0}}{r}\right)^{2(D-3)} \\
& c_{D}=\frac{D-3}{D-2} .
\end{aligned}
$$

In these so called Eddington-Finkelstein coordinates the advantages and disadvantages of the Schwarzschild coordinate system are reversed. The black hole metric is now smooth at the future event horizon. However in the limit $r \rightarrow \infty$ the spacetime metric $d s^{2}=$ $2 d v d r-d v^{2}+r^{2} d \Omega_{D-2}^{2}$ is not manifestly Minkowskian. 
The further coordinate change to the 'Kerr-Schild' time coordinate $t$ is specified by

$$
d v=d t+d r
$$

It is easy to see that the Kerr-Schild time $t$ agrees with the Schwarzschild time coordinate at large $r$, but effectively reduces to the Eddington-Finkelstein time coordinate at the first zero of $f(r)$ (when approached from large $r$ ), i.e. at the outer event horizon. For this reason one might anticipate that the black hole solution in Kerr-Schild coordinates is both manifestly Minkowskian at large $r$ as well as manifestly smooth at the outer future event horizon. A glance at the explicit black hole solution, (2.1) is sufficient to convince oneself that this is indeed the case.

\section{B Relating the geometric form of the metric and gauge field with the answer found in explicit computation}

In this appendix we shall present how the different structures and functions appearing in section 4 are related to the functions and data appearing in subsection 3.13 (the explicit computation with $\mathrm{SO}(d+1)$ invariance).

As explained in subsection 3.3, for explicit computation we assumed the following metric for the flat space-time.

$$
d s_{\text {flat }}^{2}=\eta_{M N} d x^{M} d x^{N}=\eta_{\mu \nu} d x^{\mu} d x^{\nu}+S^{2} \Omega_{i j} d \theta^{i} d \theta^{j},
$$

where $\eta_{\mu \nu} d x^{\mu} d x^{\nu}=d w_{a} d w^{a}+d S^{2}$ is the metric in the auxiliary space of $\left\{x^{\mu}\right\}$ (see subsection 3.3.4) and $\Omega_{i j}$ is the metric of a $d$ dimensional unit sphere $\left(\left\{\theta^{i}\right\}\right.$ are the angular coordinates along the isometry directions). Now because of the isometry the geometric forms will have the following properties.

1. For any geometric vector $\mathfrak{V}_{M}$, the components along the $\left\{\theta^{i}\right\}$ directions will be zero (i.e., $\mathfrak{V}_{\theta^{i}}=0$ ).

2. Similarly for any geometric tensor $\mathfrak{H}_{M N}$

$$
\mathfrak{H}_{\mu \theta^{i}}=0 \text { and } \mathfrak{H}_{\theta^{i} \theta^{j}} \propto \Omega_{i j} .
$$

3. As explained before, apart from $n_{\mu}$ and $u_{\mu}$ there is one more special vector in the auxiliary space: $Z_{M} d x^{M}=Z_{\mu} d x^{\mu}=\frac{d S}{S}-\left(\frac{n_{S}}{S}\right) n_{\mu} d x^{\mu}$. Using these $Z$ one-form we can further decompose any geometric vector and tensor scalar, vector and tensor of the $\mathrm{SO}(p)$ isometry group in the $(p+3)$ dimensional auxiliary space.

Using these properties we can translate the results in the geometric form to the language of 'auxiliary space'. For most of the functions, the translation is straightforward and we present the dictionary in table 3 and table 4 . In table 3 we present how the three scalar, two vector and one tensor function appearing in the geometric form of the metric and gauge field ((4.1)) decompose into seven scalar, three vector and one tensor function appearing 


\begin{tabular}{|c|c|}
\hline Explicit Computation & Geometric Form \\
\hline$\left(\frac{1}{D}\right) S_{(V V)}$ & $H^{(S)}+\mathcal{O}\left(\frac{1}{D}\right)^{2}$ \\
\hline$\left(\frac{1}{D}\right) S_{(A V)}$ & $A^{(S)}+\mathcal{O}\left(\frac{1}{D}\right)^{2}$ \\
\hline$\left(\frac{1}{D}\right) S_{(V z)}$ & $Z^{\mu} H_{\mu}^{(V)}+\mathcal{O}\left(\frac{1}{D}\right)^{2}$ \\
\hline$\left(\frac{1}{D}\right) S_{(z z)}$ & $Z^{\mu} Z^{\nu} H_{\mu \nu}^{(T)}+\mathcal{O}\left(\frac{1}{D}\right)^{2}$ \\
\hline$\left(\frac{1}{D}\right) S_{(A z)}$ & $Z^{\mu} A_{\mu}^{(V)}+\mathcal{O}\left(\frac{1}{D}\right)^{2}$ \\
\hline$\left(\frac{1}{D}\right) S_{(T r)}$ & $\left(\frac{1}{p}\right) P^{\mu \nu} H_{\mu \nu}^{(T)}+H^{(T r)}+\mathcal{O}\left(\frac{1}{D}\right)^{2}$ \\
\hline$\left(\frac{1}{D}\right) V_{\mu}^{(V)}$ & $P_{\mu}^{\nu} H_{\nu}^{(V)}+\mathcal{O}\left(\frac{1}{D}\right)^{2}$ \\
\hline$\left(\frac{1}{D}\right) V_{\mu}^{(z)}$ & $P_{\mu}^{\alpha} Z^{\beta} H_{\alpha \beta}^{(T)}+\mathcal{O}\left(\frac{1}{D}\right)^{2}$ \\
\hline$\left(\frac{1}{D}\right) V_{\mu}^{(A)}$ & $P_{\mu}^{\nu} A_{\nu}^{(V)}+\mathcal{O}\left(\frac{1}{D}\right)^{2}$ \\
\hline$\left(\frac{1}{D}\right) T^{\mu \nu}$ & $P^{\mu \alpha} P^{\nu \beta}\left[H_{\alpha \beta}^{(T)}-\left(\frac{\mathfrak{g}_{\alpha \beta}}{p}\right) P^{\nu_{1} \nu_{2}} H_{\nu_{1} \nu_{2}}^{(T)}\right]+\mathcal{O}\left(\frac{1}{D}\right)^{2}$ \\
\hline
\end{tabular}

Table 3. Here we relate how the functions appearing in equation (3.24) are related to the geometric form of the metric and the gauge field as in equation (4.1).

in equation $((3.24))$. Then in table 4 we decompose the geometric data in terms of the non-geometric ones (with $\mathrm{SO}(d+1)$ invariance) used for explicit computation.

However for for $\delta \phi$, i.e. the fluctuation in the radius of the $d$ dimensional sphere, the translation rule becomes a bit more subtle to be presented in a table. For convenience we shall explain it separately in subsection B.1.

Using the tables 3 and 4 and the argument presented in subsection B.1 we could easily see that if we specialize the metric and the gauge field as presented in equations (4.1), (4.2), (4.3) and (4.4) to $\mathrm{SO}(d+1)$ isometry (where $d=D-p-3$ ), they indeed reduce to the explicit solution presented in subsection 3.13 upto correction of $\mathcal{O}\left(\frac{1}{D}\right)^{2}$.

\section{B.1 Relating $\delta \phi$ to the geometric forms}

Note that any geometric tensor will have some nonzero components along the isometry directions and also because of symmetry the components must be proportional to the metric of the $d$ dimensional unit sphere. We can explicitly compute this proportionality factor which will be directly related to $\delta \phi$.

Consider the traceless tensor $H_{M N}^{(T)}$ appearing in equation (4.1) and suppose $H_{\theta^{i} \theta^{j}}^{(T)}=$ $S^{2} \mathfrak{H} \Omega_{i j}$ where $\mathfrak{H}$ is some scalar function. Then it follows that

$$
\begin{aligned}
0 & =\eta^{M N} H_{M N}^{(T)}=\eta^{\mu \nu} H_{\mu \nu}^{(T)}+\frac{\Omega^{i j}}{S^{2}} H_{\theta^{i} \theta^{j}}^{(T)}=\eta^{\mu \nu} H_{\mu \nu}^{(T)}+d \times \mathfrak{H} \\
\Rightarrow \mathfrak{H} & =-\frac{\eta^{\mu \nu} H_{\mu \nu}^{(T)}}{D-p-3} .
\end{aligned}
$$




\begin{tabular}{|c|c|}
\hline Geometric data & Data used in computation \\
\hline $\mathfrak{S}_{(1)}$ & $\left(\frac{S}{n_{S}}\right)\left[\frac{\mathfrak{s}^{(5)}}{Q}-\mathfrak{s}^{(1)}+\left(\frac{S}{n_{S}}\right) \mathfrak{s}^{(2)}\right]$ \\
\hline $\mathfrak{S}_{(2)}$ & $\left(\frac{S}{n_{S}}\right)\left[\mathfrak{s}^{(1)}-\left(\frac{S}{n_{S}}\right) \mathfrak{s}^{(2)}\right]$ \\
\hline$Z_{\mu} \mathfrak{V}_{(1)}^{\mu}$ & $\left(\frac{S}{n_{S}}\right)^{2} \mathfrak{s}^{(6)}+\mathfrak{s}^{(1)}-\left(\frac{1-n_{S}^{2}}{S \times n_{S}}\right)^{2}$ \\
\hline$Z_{\mu} \mathfrak{V}_{(2)}^{\mu}$ & $\left(\frac{S}{n_{S}}\right)^{2} \mathfrak{s}^{(6)}-\mathfrak{s}^{(1)}-\left(\frac{1-n_{S}^{2}}{S \times n_{S}}\right)^{2}$ \\
\hline$Z_{\mu} Z_{\nu} \mathfrak{T}^{\mu \nu}$ & $\mathfrak{s}^{(6)}-\left(\frac{n_{S}}{S}\right) \mathfrak{s}^{(2)}$ \\
\hline$P_{\mu \nu} \mathfrak{T}^{\mu \nu}$ & $\mathfrak{s}^{(3)}-\mathfrak{s}^{(4)}$ \\
\hline$P_{\mu \nu} \mathfrak{V}_{(1)}^{\nu}$ & $\left(\frac{S}{n_{S}}\right)^{2}\left[\mathfrak{v}_{\mu}^{(5)}+\left(\frac{n_{S}}{S}\right) \mathfrak{v}_{\mu}^{(2)}\right]$ \\
\hline$P_{\mu \nu} \mathfrak{V}_{(2)}^{\nu}$ & $\left(\frac{S}{n_{S}}\right)^{2}\left[\mathfrak{v}_{\mu}^{(5)}-\left(\frac{n_{S}}{S}\right) \mathfrak{v}_{\mu}^{(2)}\right]$ \\
\hline$Z_{\nu} P_{\alpha \mu} \mathfrak{T}^{\alpha \nu}$ & $\mathfrak{v}_{\mu}^{(5)}-\mathfrak{v}_{\mu}^{(3)}-\left(\frac{n_{S}}{S}\right) \mathfrak{v}_{\mu}^{(1)}$ \\
\hline
\end{tabular}

Table 4. Decomposition of geometric data in the special case of $\mathrm{SO}(d+1)$ symmetry in terms of the data in auxiliary space used for explicit computation.

Similarly consider the tensor $H^{(T r)} \mathcal{P}_{M N}$ appearing in (4.1)). Since we know that $n_{\theta^{i}}=$ $u_{\theta^{i}}=0$, the nonzero components of this tensor along the isometry directions are simply given by

$$
H^{(T r)} \mathcal{P}_{\theta^{i} \theta^{j}}=H^{(T r)} S^{2} \Omega_{i j}
$$

From equation (B.1) and (B.2) and the definition of $\delta \phi$ (recall that the fluctuation in the radius of the $d$ dimensional sphere $\left.=S^{2} \delta \phi\right)$, it follows that

$$
\delta \phi=H^{(T r)}+\mathfrak{H}=H^{(T r)}-\left(\frac{1}{D-p-3}\right) \eta^{\mu \nu} H_{\mu \nu}^{(T)} .
$$

The second term in the r.h.s. of equation (B.3) is of $\mathcal{O}\left(\frac{1}{D}\right)^{2}$ since by construction $H_{\mu \nu}^{(T)}$ starts at $\mathcal{O}\left(\frac{1}{D}\right)$. Now from explicit computation we know that $\delta \phi$ is of $\mathcal{O}\left(\frac{1}{D}\right)^{2}$ (see equation (3.57)) . Then it immediately follows that $H^{(T r)}$ also must start from terms of $\mathcal{O}\left(\frac{1}{D}\right)^{2}$.

We could explicitly compute the second term in r.h.s. of (B.3) in terms of the functions appearing in equation (3.24). Note that $\eta^{\mu \nu}$ could be expanded as

$$
\eta^{\mu \nu}=n^{\mu} n^{\nu}-u^{\mu} u^{\nu}+\frac{S^{2}}{1-n_{S}^{2}} Z^{\mu} Z^{\nu}+P^{\mu \nu}+\mathcal{O}\left(\frac{1}{D}\right) .
$$

Using this expansion of $\eta^{\mu \nu}$ and the translation rules as given in table 3 we find

$$
\left(\frac{1}{D-p-3}\right) \eta^{\mu \nu} H_{\mu \nu}^{(T)}=\frac{1}{D^{2}}\left[\left(\frac{1-n_{S}^{2}}{S^{2}}\right) S_{z z}+p \times S_{(T r)}\right]+\mathcal{O}\left(\frac{1}{D}\right)^{3} .
$$


In equation (B.4) we have used the fact that $H^{(T r)}$ is of $\mathcal{O}\left(\frac{1}{D}\right)^{2}$ and by construction $u^{\mu} H_{\mu \nu}^{(T)}=n^{\mu} H_{\mu \nu}^{(T)}=0$. Substituting equation (B.4) in equation (B.3) we found that

$$
H^{(T r)}=\frac{1}{D^{2}}\left[\delta \phi^{(2)}+\left(\frac{1-n_{S}^{2}}{S^{2}}\right) S_{z z}+p \times S_{(T r)}\right]+\mathcal{O}\left(\frac{1}{D}\right)^{3} .
$$

Now from equation (3.57) it directly follows that

$$
H^{(T r)}=\mathcal{O}\left(\frac{1}{D}\right)^{3}
$$

\section{Relating equations of motion expressed in different forms}

In this section we shall first state a set of algebraic identities that are true upto corrections of $\mathcal{O}\left(\frac{1}{D}\right)$. Using these identities we could easily show that the equations of motion as derived in subsection $3.10((3.37)$ and (3.39)) are equivalent to equations (4.7) and (4.8).

1. Identity-1:

$$
\begin{array}{rlrl}
\tilde{V}_{\perp} \cdot K \cdot u & =u \cdot K \cdot \tilde{V}_{\perp}=[(u \cdot \partial) n] \cdot \tilde{V}_{\perp} & \\
& =-\left[(u \cdot \partial) \tilde{V}_{\perp}\right] \cdot n & & \text { Since } n \cdot \tilde{V}_{\perp}=0 \\
& =\left(\frac{n_{S}}{S}\right)[(u \cdot \partial) u] \cdot n+(u \cdot \partial)\left(\frac{n_{S}}{S}\right)+\mathcal{O}\left(\frac{1}{D}\right) & & \text { Since } u \cdot d S=\mathcal{O}\left(\frac{1}{D}\right) \\
& =-\left(\frac{n_{S}}{S}\right)(u \cdot K \cdot u)+(u \cdot \partial)\left(\frac{n_{S}}{S}\right)+\mathcal{O}\left(\frac{1}{D}\right) & & \text { Since } u \cdot n=0 .
\end{array}
$$

Here $\tilde{V}_{\perp}=Z-\left(\frac{n_{S}}{S}\right) u=\frac{d S}{S}-\left(\frac{n_{S}}{S}\right)(n+u)=\left(\frac{S}{n_{S}}\right)(X-u)$.

2. Identity-2:

$$
\begin{aligned}
\tilde{V}_{\perp} \cdot \partial u \cdot Z & =-\tilde{V}_{\perp} \cdot \partial Z \cdot u+\mathcal{O}\left(\frac{1}{D}\right) \quad \text { Since } u \cdot Z=\mathcal{O}\left(\frac{1}{D}\right) \\
& =\left(\frac{n_{S}}{S}\right) \tilde{V}_{\perp} \cdot K \cdot u+\mathcal{O}\left(\frac{1}{D}\right) \quad \text { Since } u \cdot d S=\mathcal{O}\left(\frac{1}{D}\right), u \cdot n=0 .
\end{aligned}
$$

3. Identity-3:

$$
\begin{aligned}
u \cdot \partial u \cdot Z & =-u \cdot \partial Z \cdot u+\mathcal{O}\left(\frac{1}{D}\right) \quad \text { Since } u \cdot Z=\mathcal{O}\left(\frac{1}{D}\right) \\
& =\left(\frac{n_{S}}{S}\right) u \cdot K \cdot u+\mathcal{O}\left(\frac{1}{D}\right) \quad \text { Since } u \cdot d S=\mathcal{O}\left(\frac{1}{D}\right), \quad u \cdot n=0
\end{aligned}
$$

4. Identity-4:

$$
\begin{aligned}
\left(\frac{S}{n_{S}}\right) & \tilde{V}_{\perp} \cdot \partial(u-n) \cdot Z \\
= & \tilde{V}_{\perp} \cdot K \cdot u-\left(\frac{S}{n_{S}}\right) \tilde{V}_{\perp} \cdot K \cdot Z+\mathcal{O}\left(\frac{1}{D}\right) \quad \text { Using (C.2) } \\
& =-\left(\frac{S}{n_{S}}\right) \tilde{V}_{\perp} \cdot K \cdot \tilde{V}_{\perp}+\mathcal{O}\left(\frac{1}{D}\right) \quad \text { Since } Z=\tilde{V}_{\perp}+\frac{n_{S}}{S} u .
\end{aligned}
$$


5. Identity-5:

$$
\begin{aligned}
{[u \cdot \partial u} & \left.-\left(\frac{S}{n_{S}}\right) Z \cdot \partial n\right] \cdot Z \\
& =\left(\frac{n_{S}}{S}\right) u \cdot K \cdot u-\left(\frac{S}{n_{S}}\right) Z \cdot K \cdot Z+\mathcal{O}\left(\frac{1}{D}\right) \quad \text { Using }(\text { C.3 }) \\
& =-\left(\frac{S}{n_{S}}\right) \tilde{V}_{\perp} \cdot K \cdot \tilde{V}_{\perp}-2(Z \cdot K \cdot u)+2\left(\frac{n_{S}}{S}\right) u \cdot K \cdot u+\mathcal{O}\left(\frac{1}{D}\right) \\
& =-\left(\frac{S}{n_{S}}\right) \tilde{V}_{\perp} \cdot K \cdot \tilde{V}_{\perp}-2\left(\tilde{V}_{\perp} \cdot K \cdot u\right)+\mathcal{O}\left(\frac{1}{D}\right) .
\end{aligned}
$$

Using (C.4) and (C.5) we could very easily compute the projection of (4.7) along $Z$ direction. It turns out to be the following,

$$
\begin{aligned}
0 & =\left[(u-X) \cdot \partial O-Q^{2}(u \cdot \partial) u-Q^{2}(X \cdot K)\right] \cdot Z+\left(\frac{n_{S}}{S}\right)\left(1-Q^{2}\right)(X \cdot Z) \\
& =-\left(1-Q^{2}\right)\left[\left(\frac{S}{n_{S}}\right) \tilde{V}_{\perp} \cdot K \cdot \tilde{V}_{\perp}-Z \cdot Z\right]+2 Q^{2}\left(\tilde{V}_{\perp} \cdot K \cdot u\right)+\mathcal{O}\left(\frac{1}{D}\right) \\
& =-\left(\frac{S}{n_{S}}\right)\left(1-Q^{2}\right)\left[\tilde{V}_{\perp} \cdot K \cdot \tilde{V}_{\perp}-\left(\frac{n_{S}\left(1-n_{S}^{2}\right)}{S^{3}}\right)\right]+2 Q^{2}\left(\tilde{V}_{\perp} \cdot K \cdot u\right)+\mathcal{O}\left(\frac{1}{D}\right) .
\end{aligned}
$$

Equation (C.6) is simply equal to $\left[-\frac{S}{n_{S}}\left(1-Q^{2}\right)\right]$ times the first equation in (3.37). Second equation of (3.37) follows once we substitute (C.1) in equation (4.8).

\section{Notation and translation}

Through this paper we have had occasion to work with functions (like $\rho$ and $Q$ ) and oneform or vector fields (like $u$ and $n=\frac{d \rho}{\mid d \rho}$ ) that live in flat $D$ dimensional space. We also often deal with functions and one-forms that live on the the membrane world volume. Through the paper we use the dummy indices $M, N \ldots$ to denote coordinates in the embedding flat $D$ dimensional spacetime, and the indices $A, B \ldots$ to denote coordinates on the membrane world volume. $M, N$ indices run over $D$ values, while $A, B$ indices run over $D-1$ values.

In the computational part of this paper we have assumed that our spacetimes and membrane world volumes both preserve an $\mathrm{SO}(D-p-2)$ isometry group. It follows that the spacetime metric takes the form

$$
d s^{2}=g_{\mu \nu} d x^{\mu} d x^{\nu}+e^{\phi} d \Omega_{d}^{2},
$$

where $\mu, \nu$ run over $p+3$ values and $g_{\mu \nu}$ and $\phi$ are functions only of $x^{\mu}$. We will often use the notation

$$
e^{\phi}=S^{2} .
$$

In a similar manner the metric on the membrane world volume takes the form

$$
d s^{2}=g_{a b} d x^{a} d x^{b}+e^{\phi} d \Omega_{d}^{2},
$$


where $a, b$ run over $p+2$ values. As all spacetime vector and scalar fields also preserve $\mathrm{SO}(d+1)$, for computational purposes it is sometimes useful to view these fields as living on the reduced $p+3$ dimensional manifold

$$
d s^{2}=g_{\mu \nu} d x^{\mu} d x^{\nu}
$$

(in the case of bulk fields) and

$$
d s^{2}=g_{a b} d x^{a} d x^{b}
$$

(in the case of fields that live on the membrane world volume). We use the symbols $\nabla_{M}$ and $\nabla_{A}$ to denote covariant derivatives on all of spacetime (or all of the membrane world volume) and $\tilde{\nabla}_{\mu}$ and $\tilde{\nabla}_{a}$ to denote fields on reduced spacetime (or the reduced membrane world volume).

Consider a vector field $v^{M}$ defined on all of flat space. If we assume that $v^{M}$ preserves $\mathrm{SO}(d)$ invariance, it is easily verified that

$$
\nabla_{M} v^{M}=d \frac{v \cdot \tilde{\nabla} S}{S}+\tilde{\nabla}_{\mu} v^{\mu}
$$

In a similar manner, if $v^{A}$ is a vector field on the membrane then

$$
\nabla_{A} v^{A}=d \frac{v \cdot \tilde{\nabla} S}{S}+\tilde{\nabla}_{a} v^{a}
$$

If $\psi$ is a scalar field in spacetime or on the membrane then it is easily verified that

$$
\nabla^{2} \psi=d \frac{\tilde{\nabla} S . \tilde{\nabla} \psi}{S}+\tilde{\nabla}^{2} \psi
$$

(where $d S$ is regarded as a one-form in either spacetime or on the membrane depending on the space on which $\nabla^{2}$ is evaluated). Note that $d S$ on the membrane world volume is simply $d S$ in spacetime, projected onto the membrane world volume.

Finally if $v$ is a vector field in either spacetime or the membrane world volume then

$$
\nabla^{2} v=d\left(\frac{\tilde{\nabla} S \cdot \tilde{\nabla} v}{S}-d S v \cdot \tilde{\nabla} S\right)+\tilde{\nabla}^{2} v
$$

To end this section we note that the extrinsic curvature tensor for the membrane world volume takes the form

$$
K_{A B}=\left(K_{\mu \nu}, \frac{n^{S}}{S} \Omega_{i j}\right)
$$

where $\theta^{i}$ are angles on the unit $d$ sphere and $\Omega_{i j}$ is a metric on this space.

\section{E Eigenvalues of the Laplacian for vector spherical harmonics}

In this appendix we evaluate the eigenvalue of the Laplacian acting on the $l^{\text {th }}$ vector spherical harmonic. This spherical harmonic was defined in terms of the restriction of a collection of vector valued monomials to the unit sphere in subsection 5.2. 
Our strategy is to evaluate the Laplacian of $V$ - viewed as a vector valued monomial in $R^{D-1}$ - in spherical polar coordinates, and use the fact that this Laplacian vanishes (see subsection 5.2) to evaluate the Laplacian of the same vector field restricted to the unit sphere.

Consider any divergenceless vector field on $R^{D-1}$ with vanishing radial component, i.e. $V_{r}=0$. Using explicit expressions for the Christoffel symbols for flat space in polar coordinates we find

$$
\begin{aligned}
& \nabla_{r} V_{r}=0, \\
& \nabla_{r} V_{a}=\partial_{r} V_{a}-\frac{V_{a}}{r}, \\
& \nabla_{a} V_{r}=\frac{V_{a}}{r}, \\
& \nabla_{a} V_{b}=\hat{\nabla}_{a} V_{b},
\end{aligned}
$$

where $\hat{\nabla}$ denotes the covariant derivative taken on a unit sphere.

We will now use these results to evaluate $\nabla^{2} V$ on $R^{D-1}$ in spherical polar coordinates. The result of this computation depends on the free index in this equation. Let us first consider the case with the free index equal to $r$. In this case

$$
\begin{aligned}
\nabla^{2} V_{r} & =\nabla_{r}\left(\nabla_{r} V_{r}\right)+\frac{1}{r^{2}} g^{a b} \nabla_{a} \nabla_{b} V_{r}, \\
& =\frac{1}{r^{2}} \hat{\nabla}_{a} \hat{\nabla}^{a} V_{r}-\frac{1}{r^{2}} \hat{\nabla}_{a} V^{a}, \\
& =0 .
\end{aligned}
$$

In other words the vanishing of the $r$ component of $\nabla^{2} V$ is just a triviality - it follows as an identity upon assuming $V_{r}=0$ and $\nabla \cdot V=0$.

Let us now turn to the more interesting case of the free index being an angular direction on the unit sphere. In this case

$$
\begin{aligned}
\nabla^{2} V_{c}= & \nabla_{r}\left(\nabla_{r} V_{c}\right)+\frac{1}{r^{2}} g^{a b} \nabla_{a} \nabla_{b} V_{c} \\
= & \partial_{r}\left(\partial_{r} V_{c}-\frac{V_{c}}{r}\right)-\Gamma_{r c}^{a}\left(\partial_{r} V_{a}-\frac{V_{a}}{r}\right)+\frac{1}{r^{2}} \hat{\nabla}_{a} \hat{\nabla}^{a} V_{c} \\
& +\Gamma_{a r}^{a}\left(\partial_{r} V_{c}-\frac{V_{c}}{r}\right)+\frac{1}{r^{2}} \Gamma_{a c}^{r} \frac{V^{a}}{r}, \\
= & \partial_{r}\left(\partial_{r} V_{c}-\frac{V_{c}}{r}\right)-\frac{1}{r}\left(\partial_{r} V_{c}-\frac{V_{c}}{r}\right)+\frac{1}{r^{2}} \hat{\nabla}_{a} \hat{\nabla}^{a} V_{c} \\
& +\frac{D-2}{r}\left(\partial_{r} V_{c}-\frac{V_{c}}{r}\right)-\frac{V_{c}}{r^{2}} .
\end{aligned}
$$

Let us now specialize to $V_{c}$ is the vector field corresponding to the $l^{\text {th }}$ vector spherical harmonic. In this case $V_{c} \propto r^{l+1}$. Using this fact and $\nabla^{2} V_{c}=0$ we get

$$
-\hat{\nabla}^{2} V_{c}=(l(l+1)-l-l+(D-2) l-1) V_{c}=[(D+l-3) l-1] V_{c} .
$$


Open Access. This article is distributed under the terms of the Creative Commons Attribution License (CC-BY 4.0), which permits any use, distribution and reproduction in any medium, provided the original author(s) and source are credited.

\section{References}

[1] S. Bhattacharyya, A. De, S. Minwalla, R. Mohan and A. Saha, A membrane paradigm at large $D$, arXiv:1504.06613 [inSPIRE].

[2] R. Emparan, R. Suzuki and K. Tanabe, The large D limit of general relativity, JHEP 06 (2013) 009 [arXiv: 1302.6382] [INSPIRE].

[3] R. Emparan, D. Grumiller and K. Tanabe, Large-D gravity and low-D strings, Phys. Rev. Lett. 110 (2013) 251102 [arXiv:1303.1995] [INSPIRE].

[4] R. Emparan and K. Tanabe, Holographic superconductivity in the large D expansion, JHEP 01 (2014) 145 [arXiv: 1312.1108] [INSPIRE].

[5] R. Emparan and K. Tanabe, Universal quasinormal modes of large D black holes, Phys. Rev. D 89 (2014) 064028 [arXiv: 1401.1957] [INSPIRE].

[6] R. Emparan, R. Suzuki and K. Tanabe, Instability of rotating black holes: large D analysis, JHEP 06 (2014) 106 [arXiv:1402.6215] [INSPIRE].

[7] R. Emparan, R. Suzuki and K. Tanabe, Decoupling and non-decoupling dynamics of large D black holes, JHEP 07 (2014) 113 [arXiv:1406.1258] [INSPIRE].

[8] R. Emparan, R. Suzuki and K. Tanabe, Quasinormal modes of (Anti-)de Sitter black holes in the $1 / D$ expansion, JHEP 04 (2015) 085 [arXiv: 1502.02820] [INSPIRE].

[9] R. Emparan, T. Shiromizu, R. Suzuki, K. Tanabe and T. Tanaka, Effective theory of black holes in the $1 / D$ expansion, JHEP 06 (2015) 159 [arXiv: 1504.06489] [INSPIRE].

[10] R. Suzuki and K. Tanabe, Stationary black holes: large D analysis, JHEP 09 (2015) 193 [arXiv: 1505.01282] [INSPIRE].

[11] R. Emparan, R. Suzuki and K. Tanabe, Evolution and end point of the black string instability: large D solution, Phys. Rev. Lett. 115 (2015) 091102 [arXiv:1506.06772] [INSPIRE].

[12] R. Suzuki and K. Tanabe, Non-uniform black strings and the critical dimension in the $1 / D$ expansion, JHEP 10 (2015) 107 [arXiv:1506.01890] [INSPIRE].

[13] K. Tanabe, Black rings at large D, JHEP 02 (2016) 151 [arXiv:1510.02200] [INSPIRE].

[14] S. Bhattacharyya et al., Charged Stationary black holes from membranes, (2014).

[15] S. Bhattacharyya, V.E. Hubeny, S. Minwalla and M. Rangamani, Nonlinear fluid dynamics from gravity, JHEP 02 (2008) 045 [arXiv: 0712.2456] [INSPIRE].

[16] J. Erdmenger, M. Haack, M. Kaminski and A. Yarom, Fluid dynamics of R-charged black holes, JHEP 01 (2009) 055 [arXiv:0809.2488] [INSPIRE].

[17] N. Banerjee et al., Hydrodynamics from charged black branes, JHEP 01 (2011) 094 [arXiv: 0809.2596] [INSPIRE].

[18] S. Bhattacharyya, R. Loganayagam, I. Mandal, S. Minwalla and A. Sharma, Conformal nonlinear fluid dynamics from gravity in arbitrary dimensions, JHEP 12 (2008) 116 [arXiv:0809.4272] [INSPIRE]. 
[19] M. Rangamani, Gravity and hydrodynamics: lectures on the fluid-gravity correspondence, Class. Quant. Grav. 26 (2009) 224003 [arXiv:0905.4352] [INSPIRE].

[20] V.E. Hubeny, S. Minwalla and M. Rangamani, The fluid/gravity correspondence, arXiv: 1107.5780 [INSPIRE].

[21] R.C. Myers and M.J. Perry, Black holes in higher dimensional space-times, Annals Phys. 172 (1986) 304 [INSPIRE].

[22] H. Kodama and A. Ishibashi, Master equations for perturbations of generalized static black holes with charge in higher dimensions, Prog. Theor. Phys. 111 (2004) 29 [hep-th/0308128] [INSPIRE]. 